\title{
Flight Loads Analysis with Inertially Coupled Equations of Motion
}

\author{
Christian Reschke* \\ DLR German Aerospace Center, Institute of Robotics and Mechatronics \\ Wessling 82234, Germany
}

In this work an approach for simulation of a large passenger aircraft with high precision equations of motion and a new method of dynamic loads calculation is presented, which can be used for maneuver and gust loads analysis in the time domain.

Equations of motion and equations of structural loads are derived from first principles. The consistent set of equations includes all inertial coupling terms and is tailored for direct integration of finite element models.

A dynamic simulation of a large transport aircraft is used to show the influence of inertial coupling terms on simulation and loads computation.

\section{Nomenclature}

\begin{tabular}{|c|c|c|c|}
\hline Symbols & & $\psi$ & heading angle \\
\hline e & unit vector & $\theta$ & pitch attitude angle \\
\hline B & damping matrix & $\eta_{E}$ & generalized elastic coordinate \\
\hline $\begin{array}{l}\mathbf{D} \\
\mathbf{d}_{i}\end{array}$ & $\begin{array}{l}\text { transformation matrix for angular rates } \\
\text { elastic deformation in l.r.f }\end{array}$ & $\Omega_{b}$ & angular velocity of the body frame resolved in \\
\hline $\mathbf{D}_{j k}$ & substantial differentiation matrix & $\Theta$ & $\begin{array}{l}\text { body axes } \\
\text { vector of euler angles }\end{array}$ \\
\hline $\mathbf{F}_{i}$ & local force vector & $\zeta$ & modal damping parameter \\
\hline$\stackrel{\text { g }}{\mathbf{H}}$ & $\begin{array}{l}\text { gravitation vector } \\
\text { momentum }\end{array}$ & \multicolumn{2}{|c|}{ Abbreviations } \\
\hline I & identity matrix & c.g. & center of gravity \\
\hline $\mathbf{J}_{E}$ & $\begin{array}{l}\text { local inertia tensor contribution to total inertia } \\
\text { tensor }\end{array}$ & $\begin{array}{l}F S M \\
\text { l.r.f. }\end{array}$ & $\begin{array}{l}\text { Force Summation Method } \\
\text { local reference frame }\end{array}$ \\
\hline $\mathbf{J}_{i}$ & $\begin{array}{l}\text { local inertia tensor w.r.t. the location of the } \\
\text { lumped mass }\end{array}$ & w.r.t. & with respect to \\
\hline $\mathbf{J}_{S}$ & Steiner contribution to total inertia tensor & & Equation of Motion \\
\hline K & stiffness matrix & \multicolumn{2}{|c|}{ Subscripts } \\
\hline M & mass matrix & 0 & related to center of gravity \\
\hline $\mathbf{M}_{i}$ & local moment vector & $b$ & body reference frame \\
\hline $\begin{array}{l}\mathrm{Q} \\
\mathrm{Q}\end{array}$ & $\begin{array}{l}\text { aerodynamic influence coefficient matrix } \\
\text { generalized force }\end{array}$ & $\begin{array}{l}E \\
e\end{array}$ & set of generalized elastic coordinates \\
\hline $\mathbf{R}$ & position vector & $g$ & set of physical degrees of freedom \\
\hline $\mathbf{r}_{i}$ & $\begin{array}{l}\text { position vector of grid point in body reference } \\
\text { frame }\end{array}$ & & $\begin{array}{l}\text { aerodynamic control point set } \\
\text { aerodynamic loading point set }\end{array}$ \\
\hline $\mathbf{s}_{i}$ & position vector of lumped mass element in l.r.f & kin & kinetic \\
\hline $\mathbf{S}_{k j}$ & integration matrix & $n c o$ & non conservative \\
\hline $\mathbf{T}$ & transformation matrix & pot & potential \\
\hline $\mathbf{V}_{b}$ & velocity of the body frame resolved in body axes & $R$ & rigid body mode \\
\hline E & energy & $r$ & rotational \\
\hline$g$ & gravitation constant & $t$ & translational \\
\hline $\begin{array}{l}m \\
m_{i} \\
W\end{array}$ & $\begin{array}{l}\text { total mass of the airplane } \\
\text { lumped mass } \\
\text { work }\end{array}$ & \multicolumn{2}{|c|}{$\begin{array}{l}\text { Conventions } \\
\langle(\ldots j k)\rangle \text { summation: } \sum_{j=1}^{3} \sum_{k=1}^{3}(\ldots)_{j k} \mathbf{e}_{j} \mathbf{e}_{k}^{T} \\
(\ldots) \\
(\ldots)\end{array}$} \\
\hline $\begin{array}{l}\text { Greek } S\} \\
\delta \alpha \\
\delta \\
\mathcal{L} \\
\varphi_{i} \\
\Phi \\
\phi\end{array}$ & $\begin{array}{l}\text { mbols } \\
\text { virtual angular displacements of the body frame } \\
\text { virtual variation } \\
\text { Lagrange variable } \\
\text { rot. elastic deformation in l.r.f } \\
\text { modal matrix } \\
\text { roll attitude angle angle }\end{array}$ & $\begin{array}{l}(\cdots) \\
(\ldots) \\
(\ldots) \\
\operatorname{diag}(\ldots) \\
\times \\
s k(\ldots) \\
T\end{array}$ & $\begin{array}{l}\text { time derivative w.r.t. body frame } \\
\text { time derivative w.r.t. inertial frame } \\
\text { diagonal matrix } \\
\text { vector cross product } \\
\text { skew symmetric matrix } \mathbf{a} \times \mathbf{b}=\operatorname{sk}(\mathbf{a}) \mathbf{b} \\
\text { transpose }\end{array}$ \\
\hline
\end{tabular}

*Research Engineer, AIAA Member, christian.reschke@dlr.de 


\section{Introduction}

Flight loads analysis considers structural loads due to maneuvers and atmospheric gust. Especially for large flexible aircraft, simulation models have to be capable of representing large amplitude rigid body motion and the elastic deformation of the airframe. Analysis tools integrate equations of motions (EOM), modules providing aerodynamic forces, a nonlinear electronic flight control system (EFCS) and equations for structural loads recovery (EOL).

EOM for a free flying flexible aircraft are already addressed by Bisplinghoff and Ashley. ${ }^{3}$ Etkin $^{10}$ and McLean ${ }^{16}$ augment the flight mechanics equations by elastic degrees of freedom. Waszak and Schmidt ${ }^{24}$ derive the equations of motion from first principles using Lagrange's equations. All assumptions and simplifications are clearly mentioned.

However, all of the above references assume a continuous elastic body. Structural dynamic models are usually obtained by reducing complex finite element models and including lumped masses attached to the nodes. Cavin ${ }^{6}$ includes finite element shape functions in the his formulation of EOM.

All listed references make assumptions leading to inertially decoupled equations of motion. Buttrill ${ }^{4}$ derives equations of motion assuming a lumped mass finite element model and retains all inertial coupling terms. Also Gupta and Brenner ${ }^{15}$ account for inertial coupling terms. However both references do not account for nodal rotational degrees of freedom, a drawback for integration of practical finite element models. Hanel $^{12}$ derives the equations, based on the method of Newton Euler. Nodal rotational degrees of freedom and inertial coupling are partially included.

Meirovitch ${ }^{18}$ derives Lagrange's equations for quasi-coordinates first and then derives equations of motion accounting for all inertial coupling effects. However the formulation is not tailored towards integration of lumped mass finite element models. ${ }^{19}$

For flight loads analysis based on a lumped mass finite element model the question of loads recovery arises. None of the above references, except Bisplinghoff and Ashley, ${ }^{3}$ addresses the EOL directly.

A variety of other references focus on the two most common loads recovery techniques, the mode displacement and the force summation method, also referred to as mode acceleration method. The deformation approach or Mode Displacement Method ${ }^{3,7-9,11,14,21,21}$ recovers elastic loads from nodal deformation. The force summation method, classically derived for linear aeroelastic system, ${ }^{14,20,21}$ solves the half generalized aeroelastic equation of motion for the elastic forces. Convergence studies ${ }^{3,8,14,21}$ show a superior convergence behavior of the force summation method.

Flight loads analysis tools requires the equations of motion and equations of structural loads to be based on consistent assumptions. None of the listed references provides a consistent set of EOM/EOL capable of direct finite element model integration. Especially EOL based on the force summation method do not account for combination with nonlinear inertially coupled EOM. The focus of the present work is to close the gap between available EOM and EOL formulations. Also, special attention is paid on the influence of inertial coupling on structural loads.

The present work derives the EOM from first principles in section II. Emphasize of the derivation is to arrive at a fully generalized formulation that is suitable for rapid time domain simulation. Rotational nodal degrees of freedom, mass offsets and all inertial coupling terms are accounted for in the new formulation. In section [II a consistent loads equation EOL is derived. The new formulation is the force summation method for nonlinear equations of motion with inertial coupling. Section IV] describes the modelling of the external forces subsequently used for simulation. A example, pointing out the influence of the inertial coupling terms on the simulation and loads recovery, is presented in section $\mathrm{V}$. Section VI contains conclusions. Appendix VII presents a validation of the generalized EOM formulation. 


\section{Equations of Motion}

This section describes the derivation of the equations of motion for an elastic aircraft using Lagrange's equations for quasi-coordinates. The inertial coupling between rigid body motion and elastic deformation is included in the formulation. The Lagrange's equations can be written as follows: ${ }^{18}$

$$
\begin{aligned}
\frac{\partial}{\partial t}\left(\frac{\partial \mathcal{L}}{\partial \mathbf{V}_{b}}\right)+\boldsymbol{\Omega}_{b} \times\left(\frac{\partial \mathcal{L}}{\partial \mathbf{V}_{b}}\right)-\mathbf{T}_{b e} \frac{\partial \mathcal{L}}{\partial \mathbf{R}_{0 e}} & =\mathbf{T}_{b e} \mathbf{Q}_{t} \\
\frac{\partial}{\partial t}\left(\frac{\partial \mathcal{L}}{\partial \boldsymbol{\Omega}_{b}}\right)+\mathbf{V}_{b} \times\left(\frac{\partial \mathcal{L}}{\partial \mathbf{V}_{b}}\right)+\boldsymbol{\Omega}_{b} \times\left(\frac{\partial \mathcal{L}}{\partial \boldsymbol{\Omega}_{b}}\right)-\left(\mathbf{D}^{T}\right)^{-1} \frac{\partial \mathcal{L}}{\partial \boldsymbol{\Theta}} & =\left(\mathbf{D}^{T}\right)^{-1} \mathbf{Q}_{r} \\
\frac{\partial}{\partial t}\left(\frac{\partial \mathcal{L}}{\partial \stackrel{\circ}{\eta}_{E}}\right)-\frac{\partial \mathcal{L}}{\partial \eta_{E}} & =\mathbf{Q}_{E}
\end{aligned}
$$

where the Lagrange variable $\mathcal{L}$ is defined as the difference of kinetic and potential energy: $\mathcal{L}=E_{\text {kin }}-E_{\text {pot }}$ The position of the body frame resolved in inertial coordinates $\mathbf{R}_{0 e}$ and the vector of Euler angles $\boldsymbol{\Theta}$ have the following components:

$$
\mathbf{R}_{0 e}=\left[\begin{array}{lll}
\mathbf{R}_{0 e_{x}} & \mathbf{R}_{0 e_{y}} & \mathbf{R}_{0 e_{z}}
\end{array}\right]^{T} ; \quad \boldsymbol{\Theta}=\left[\begin{array}{lll}
\phi & \theta & \psi
\end{array}\right]^{T}
$$

The quasi velocity vectors are defined as follows:

$$
\mathbf{V}_{b}=\left[\begin{array}{lll}
\mathbf{V}_{b_{x}} & \mathbf{V}_{b_{y}} & \mathbf{V}_{b_{z}}
\end{array}\right]^{T} ; \quad \boldsymbol{\Omega}_{b}=\left[\begin{array}{lll}
\boldsymbol{\Omega}_{b_{x}} & \boldsymbol{\Omega}_{b_{y}} & \boldsymbol{\Omega}_{b_{z}}
\end{array}\right]^{T}
$$

At the right hand side of the Lagrange equations the terms $\mathbf{Q}_{t}, \mathbf{Q}_{r}, \mathbf{Q}_{E}$ represent the generalized nonconservative forces resulting from the derivatives of the virtual work $\delta W_{n c o}$ due to nonconservative forces:

$$
\mathbf{Q}_{t}=\frac{\partial\left(\delta W_{n c o}\right)}{\partial \mathbf{R}_{0 e}} ; \quad \mathbf{Q}_{r}=\frac{\partial\left(\delta W_{n c o}\right)}{\partial \boldsymbol{\Theta}} ; \quad \mathbf{Q}_{E}=\frac{\partial\left(\delta W_{n c o}\right)}{\partial \eta_{E}}
$$

The kinematic relations complete the equations: ${ }^{23}$

$$
\dot{\Theta}=\mathbf{D}^{-1} \boldsymbol{\Omega}_{b} ; \quad \dot{\mathbf{R}}_{0 e}=\mathbf{T}_{b e}^{-1} \mathbf{V}_{b}
$$

where the transformation from the inertial frame into body axis $\mathbf{T}_{b e}$ is given by the series of Euler angle rotations $^{23}$ and the transformation matrix $\mathbf{D}$ between the Euler angles rates $\dot{\Theta}$ and the angular velocity vector $\boldsymbol{\Omega}_{b}$ is given by: ${ }^{23}$

$$
\mathbf{T}_{b e}=\left[\begin{array}{ccc}
1 & 0 & 0 \\
0 & \cos \phi & \sin \phi \\
0 & -\sin \phi & \cos \phi
\end{array}\right]\left[\begin{array}{ccc}
\cos \theta & 0 & -\sin \theta \\
0 & 1 & 0 \\
\sin \theta & 0 & \cos \theta
\end{array}\right]\left[\begin{array}{ccc}
\cos \psi & \sin \psi & 0 \\
-\sin \psi & \cos \psi & 0 \\
0 & 0 & 1
\end{array}\right] ; \mathbf{D}=\left[\begin{array}{ccc}
1 & 0 & -\sin \theta \\
0 & \cos \phi & \cos \theta \sin \phi \\
0 & -\sin \phi & \cos \theta \cos \phi
\end{array}\right]
$$

\section{A. Definitions and Assumptions}

The formulation will be tailored towards integration of available linear finite element models, used in loads analysis and aeroelasticity. Some assumptions can then be made for the equation development:

Assumption 1: The aircraft is described as a collection of lumped mass elements, with an associated mass $m_{i}$ and inertia tensor $\mathbf{J}_{i}$.

Assumption 2: Linear elastic theory applies.

Assumption 3: Local translational and rotational elastic deformations w.r.t. the reference shape are small.

The following coordinates frame will be used (see figure 1):

- Inertial reference frame $\left(x_{e}, y_{e}, z_{e}\right)$.

${ }^{a}$ This is the typical case in finite element models used in loads and aeroelastics. Local mass and inertia is for example defined by the MSC.Nastran CONM2 cards. 


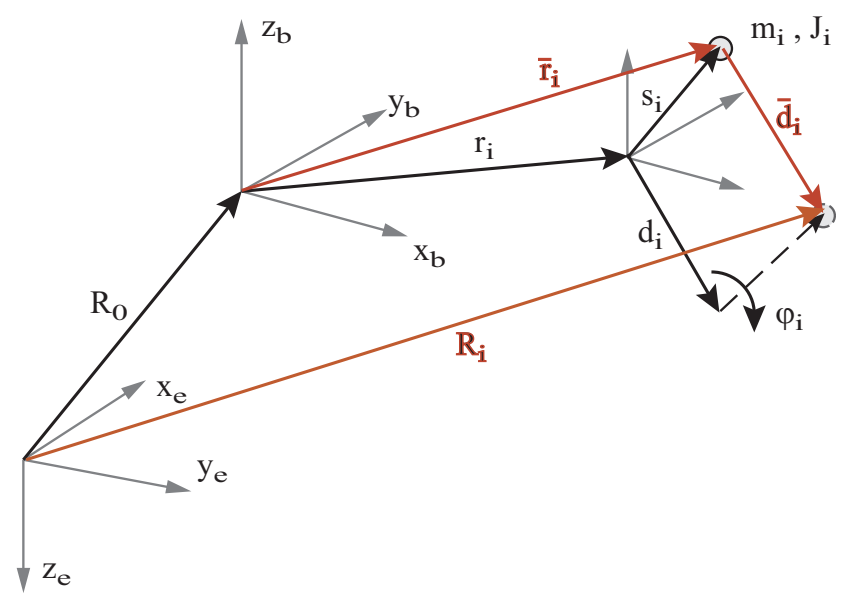

Figure 1. Location of a mass element in reference and deformed condition

- Body reference frame $\left(x_{b}, y_{b}, z_{b}\right)$ located at the momentary center of gravity of the vehicle.

- Local reference frames (l.r.f) located at the position of each grid point. The axes are assumed to be parallel to the body reference frame in undeformed shape.

The location of the local lumped mass element $m_{i}, \mathbf{J}_{i}$ resolved in the body coordinate frame may be written as follows

$$
\mathbf{R}_{i}=\mathbf{R}_{0}+\mathbf{r}_{i}+\mathbf{d}_{i}+\mathbf{T}\left(\varphi_{i}\right) \mathbf{s}_{i}
$$

where the transformation matrix $\mathbf{T}\left(\varphi_{i}\right)$ transforms the offset vector $\mathbf{s}_{i}$ in its deformed position. In accordance with assumption 3 , the linearized version of the transformation matrix, hence $\mathbf{T}\left(\varphi_{i}\right)=\mathbf{I}+s k\left(\varphi_{i}\right)$, will be used. The position of the mass element may then be written as follows

$$
\begin{aligned}
\mathbf{R}_{i} & =\mathbf{R}_{0}+\mathbf{r}_{i}+\mathbf{d}_{i}+\left(\mathbf{I}+s k\left(\varphi_{i}\right)\right) \mathbf{s}_{i} \\
& =\mathbf{R}_{0}+\overline{\mathbf{r}}_{i}+\overline{\mathbf{d}}_{i}
\end{aligned}
$$

with the vectors $\overline{\mathbf{r}}_{i}=\mathbf{r}_{i}+\mathbf{s}_{i}$ and $\overline{\mathbf{d}}_{i}=\mathbf{d}_{i}+\varphi_{i} \times \mathbf{s}_{i}$ shortening the formulation. Figure 1 shows the location of a lumped mass element $\mathbf{R}_{i}$ in its deformed condition.

The translational inertial velocity of the local mass element $m_{i}$ is then given by the time derivative of the position vector (8a) as follows:

$$
\dot{\mathbf{R}}_{i}=\mathbf{V}_{b}+\underbrace{\left(\stackrel{\circ}{\left.\mathbf{d}_{i}+\stackrel{\circ}{\varphi}_{i} \times \mathbf{s}_{i}\right)}\right.}_{\stackrel{\circ}{\mathbf{d}}_{i}}+\boldsymbol{\Omega}_{b} \times(\underbrace{\left(\mathbf{r}_{i}+\mathbf{s}_{i}\right.}_{\overline{\mathbf{r}}_{i}}+\underbrace{\left(\mathbf{d}_{i}+\varphi_{i} \times \mathbf{s}_{i}\right)}_{\mathbf{d}_{i}})
$$

where the inertial velocity $\mathbf{V}_{b}$ of the body frame (resolved in body axes) can also be written as $\mathbf{V}_{b}=\stackrel{\circ}{\mathbf{R}_{0}}$ $+\boldsymbol{\Omega}_{b} \times \mathbf{R}_{0}$ since the vector $\mathbf{R}_{0}$ is resolved in the body frame.

The rotational inertial velocity of the lumped mass element is the superposition of the rotational velocity of the body frame $\boldsymbol{\Omega}_{b}$ and the rotational velocity due to elastic deformation $\stackrel{\circ}{\varphi}_{i}$, hence:

$$
\boldsymbol{\Omega}_{i}=\boldsymbol{\Omega}_{b}+\stackrel{\circ}{\varphi}_{i}
$$

\section{B. Energy Terms}

In this section the kinetic and potential energy of the aircraft will be formulated for subsequent use in the Lagrange's equation. 


\section{Kinetic Energy}

Each mass element is defined by a lumped mass $m_{i}$ and a corresponding inertia tensor $\mathbf{J}_{i}$. Therefore the kinetic energy can be written as a contribution from the mass elements $E_{k i n, t}$ and a contribution of the local inertia tensors $E_{k i n, r}$ :

$$
E_{k i n}=\underbrace{\frac{1}{2} \sum_{i} \dot{\mathbf{R}}_{i}^{T} \dot{\mathbf{R}}_{i} m_{i}}_{E_{k i n, t}}+\underbrace{\frac{1}{2} \sum_{i} \boldsymbol{\Omega}_{i}^{T} \mathbf{J}_{i} \boldsymbol{\Omega}_{i}}_{E_{k i n, r}}
$$

The translational contribution $E_{k i n, t}$ is considered first. Expansion with the velocity expression for $\dot{\mathbf{R}}_{i}$ (9) yields

$$
\begin{aligned}
E_{k i n, t} & =\frac{1}{2} \mathbf{V}_{b}^{T} \mathbf{V}_{b} m+\frac{1}{2} \sum_{i} \stackrel{\circ}{\mathbf{d}}_{i}^{T} \stackrel{\circ}{\mathbf{d}}_{i} m_{i}+\frac{1}{2} \boldsymbol{\Omega}_{b}^{T} \underbrace{\sum_{i}\left[\left(\overline{\mathbf{r}}_{i}+\overline{\mathbf{d}}_{i}\right)^{T}\left(\overline{\mathbf{r}}_{i}+\overline{\mathbf{d}}_{i}\right) \mathbf{I}-\left(\overline{\mathbf{r}}_{i}+\overline{\mathbf{d}}_{i}\right)\left(\overline{\mathbf{r}}_{i}+\overline{\mathbf{d}}_{i}\right)^{T}\right] m_{i} \boldsymbol{\Omega}_{b}}_{\mathbf{J}_{S}} \\
& +\mathbf{V}_{b}^{T} \sum_{i} \stackrel{\circ}{\mathbf{d}_{i}} m_{i}+\mathbf{V}_{b}^{T}\left(\boldsymbol{\Omega}_{b} \times \sum_{i}\left(\overline{\mathbf{r}}_{i}+\overline{\mathbf{d}}_{i}\right) m_{i}\right)+\sum_{i}\left(\boldsymbol{\Omega}_{b} \times\left(\overline{\mathbf{r}}_{i}+\overline{\mathbf{d}}_{i}\right)\right)^{T} \stackrel{\circ}{\mathbf{d}}_{i} m_{i}
\end{aligned}
$$

where $\mathbf{J}_{S}$ represents the contribution of all mass element to the total inertial tensor of the aircraft. The last term of $(12)$ can be written as follows

$$
\sum_{i}\left(\boldsymbol{\Omega}_{b} \times\left(\overline{\mathbf{r}}_{i}+\overline{\mathbf{d}}_{i}\right)\right)^{T} \stackrel{\circ}{\stackrel{\mathbf{d}}{i}_{i} m_{i}}=\boldsymbol{\Omega}_{b}^{T} \sum_{i}\left(\overline{\mathbf{r}}_{i} \times \stackrel{\circ}{\mathbf{d}}_{i}\right) m_{i}+\boldsymbol{\Omega}_{b}^{T} \sum_{i}\left(\overline{\mathbf{d}}_{i} \times \stackrel{\circ}{\mathbf{d}}_{i}\right) m_{i}
$$

For practical mean axes constraints ${ }^{5,6,24}$ and due to placement of the origin of the body frame in the center of gravity, equation (12) with (13) simplifies to :

$$
E_{k i n, t}=\frac{1}{2} \mathbf{V}_{b}^{T} \mathbf{V}_{b} m+\frac{1}{2} \sum_{i}{\stackrel{\circ}{\mathbf{d}_{i}}}_{i}^{\frac{\circ}{\mathbf{d}_{i}}} m_{i}+\frac{1}{2} \boldsymbol{\Omega}_{b}^{T} \mathbf{J}_{S} \boldsymbol{\Omega}_{b}+\boldsymbol{\Omega}_{b}^{T} \sum_{i}\left(\overline{\mathbf{d}}_{i} \times \stackrel{\circ}{\mathbf{d}}_{i}\right) m_{i}
$$

where the last two terms represent the cross coupling between rigid body motion and elastic deformation.

The rotational contribution to kinetic energy $E_{k i n, r}$ results from the local inertia tensors and the rotational velocities of each inertia tensor. Expansion of the second term of (11) with (10) yields

$$
E_{k i n, r}=\frac{1}{2} \boldsymbol{\Omega}_{b}^{T} \underbrace{\sum_{i} \mathbf{J}_{i}}_{\mathbf{J}_{E}} \boldsymbol{\Omega}_{b}+\frac{1}{2} \sum_{i}\left\{\stackrel{\circ}{\varphi}^{T} \mathbf{J}_{i} \stackrel{\circ}{\varphi}_{i}+\stackrel{\circ}{\varphi}_{i}^{T} \mathbf{J}_{i} \boldsymbol{\Omega}_{b}+\boldsymbol{\Omega}_{b}^{T} \mathbf{J}_{i} \stackrel{\circ}{\varphi}_{i}\right\}
$$

where again the last two terms represent the cross coupling between rigid body motion and elastic deformation. It is useful to introduce the total inertia tensor of the deformed aircraft, which is given by

$$
\mathbf{J}=\mathbf{J}_{E}+\mathbf{J}_{S}
$$

With (14), (15) in (11) and (16) the total kinetic energy can be written in the following form:

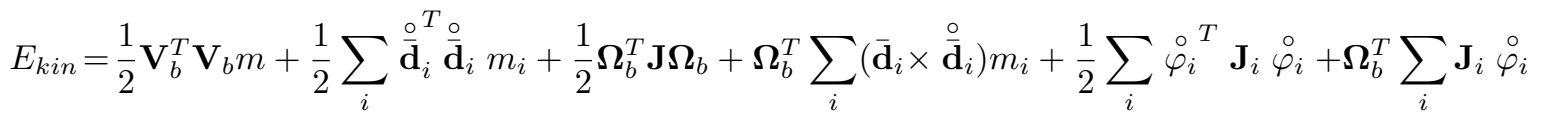

The second term $\frac{1}{2} \sum_{i} \stackrel{\circ}{\mathbf{d}}_{i}^{T} \stackrel{\circ}{\mathbf{d}}_{i} m_{i}$ of (17) can be expanded using the expression for elastic velocity of the mass element $\stackrel{\circ}{\mathbf{d}_{i}}=\stackrel{\circ}{\mathbf{d}}_{i}+\stackrel{\circ}{\varphi}_{i} \times \mathbf{s}_{i}$, defined in $(\overline{9})$, :

$$
\frac{1}{2} \sum_{i}{\stackrel{\circ}{\mathbf{d}_{i}}}_{i}^{\circ} \stackrel{\circ}{\mathbf{d}}_{i} m_{i}=\frac{1}{2} \sum_{i}\left\{\stackrel{\circ T}{\mathbf{d}_{i} \stackrel{\circ}{\mathbf{d}}_{i}+2} \stackrel{\circ}{\stackrel{\circ}{\mathbf{d}}_{i}}\left(\stackrel{\circ}{\varphi_{i}} \times \mathbf{s}_{i}\right)+\stackrel{\circ}{\varphi}_{i}^{T} \quad s k\left(\mathbf{s}_{i}\right)^{T} s k\left(\mathbf{s}_{i}\right) \stackrel{\circ}{\varphi}_{i}\right\} m_{i}
$$


Combining the second $\frac{1}{2} \sum_{i}{\stackrel{\circ}{\mathbf{d}_{i}}}_{i}^{T} \stackrel{\circ}{\mathbf{d}}_{i} m_{i}$ and the fifth term $\frac{1}{2} \sum_{i} \stackrel{\circ}{\varphi}_{i}^{T} \mathbf{J}_{i} \stackrel{\circ}{\varphi}_{i}$ of (17) and using the expression for the local inertia tensor w.r.t to the grid point i $\mathbf{J}_{g, i}=\mathbf{J}_{i}+s k\left(\mathbf{s}_{i}\right)^{T} s k\left(\mathbf{s}_{i}\right) m_{i}$ yields:

$$
\frac{1}{2} \sum_{i} \stackrel{\circ}{\mathbf{d}}_{i}^{T} \stackrel{\circ}{\mathbf{d}}_{i} m_{i}+\frac{1}{2} \sum_{i} \stackrel{\circ}{\varphi}_{i}^{T} \mathbf{J}_{i} \stackrel{\circ}{\varphi}_{i}=\frac{1}{2} \sum_{i}\left[\begin{array}{c}
\circ \\
\mathbf{d}_{i} \\
\stackrel{\varphi}{\varphi}_{i}
\end{array}\right]^{T}\left[\begin{array}{cc}
m_{i} \mathbf{I} & -m_{i} s k\left(\mathbf{s}_{i}\right) \\
m_{i} s k\left(\mathbf{s}_{i}\right) & \mathbf{J}_{g, i}
\end{array}\right]\left[\begin{array}{c}
\circ \\
\mathbf{d}_{i} \\
\check{\varphi}_{i}
\end{array}\right]
$$

The block diagonal mass matrix $\mathbf{M}_{g g}$ and a set of free-free vibration mode shapes $\boldsymbol{\Phi}_{g_{E}}$ will now be included in the formulation.

Assumption 4: Orthogonal mode shapes resulting from free-free modal analysis are available. The deformation of the airplane may be written as a linear combination of the mode shapes, i.e. the modal approach will be used.

The deformation is now written as a linear combination of the mode shapes:

$$
\left[\begin{array}{c}
\mathbf{d}_{i} \\
\varphi_{i}
\end{array}\right]=\left[\begin{array}{c}
\boldsymbol{\Phi}_{g_{i} E_{t}} \\
\boldsymbol{\Phi}_{g_{i} E_{r}}
\end{array}\right] \eta_{E}=\boldsymbol{\Phi}_{g_{i} E} \eta_{E}
$$

Hence the second and fifth term can then be simplified to

$$
\begin{aligned}
& \frac{1}{2} \stackrel{\circ}{\eta}_{E}^{T} \sum_{i}\left[\begin{array}{l}
\boldsymbol{\Phi}_{g_{i} E_{t}} \\
\boldsymbol{\Phi}_{g_{i} E_{r}}
\end{array}\right]^{T}\left[\begin{array}{cc}
m_{i} \mathbf{I} & -m_{i} s k\left(\mathbf{s}_{i}\right) \\
m_{i} s k\left(\mathbf{s}_{i}\right) & \mathbf{J}_{g, i}
\end{array}\right]\left[\begin{array}{c}
\mathbf{\Phi}_{g_{i} E_{t}} \\
\boldsymbol{\Phi}_{g_{i} E_{r}}
\end{array}\right] \stackrel{\circ}{\eta}_{E} \\
= & \frac{1}{2} \stackrel{\circ}{\eta}_{E}^{T} \boldsymbol{\Phi}_{g_{E}}^{T} \mathbf{M}_{g g} \boldsymbol{\Phi}_{g_{E}} \stackrel{\circ}{\eta}_{E} \\
= & \frac{1}{2} \stackrel{\circ}{\eta}_{E}^{T} \mathbf{M}_{E E} \stackrel{\circ}{\eta}_{E}
\end{aligned}
$$

The total kinetic energy can now be written as follows:

$$
E_{k i n}=\frac{1}{2} \mathbf{V}_{b}^{T} \mathbf{V}_{b} m+\frac{1}{2} \boldsymbol{\Omega}_{b}^{T} \mathbf{J} \boldsymbol{\Omega}_{b}+\frac{1}{2} \stackrel{\circ}{\eta}_{E}^{T} \mathbf{M}_{E E} \stackrel{\circ}{\eta}_{E}+\boldsymbol{\Omega}_{b}^{T} \sum_{i}\left(\overline{\mathbf{d}}_{i} \times \stackrel{\circ}{\mathbf{d}}_{i}\right) m_{i}+\boldsymbol{\Omega}_{b}^{T} \sum_{i} \mathbf{J}_{i} \stackrel{\circ}{\varphi}_{i}
$$

\section{Potential Energy}

The potential energy is given by

$$
E_{p o t}=-\sum_{i}\left(\mathbf{T}_{e b} \mathbf{R}_{i}\right)^{T} \mathbf{g}_{e} m_{i}+\frac{1}{2} \eta_{E}^{T} \mathbf{K}_{E E} \eta_{E}
$$

where the $\mathbf{K}_{E E}$ is the generalized stiffness matrix and $\mathbf{g}_{e}$ is the constant gravitation vector resolved in the inertial frame:

$$
\mathbf{g}_{e}=\left[\begin{array}{lll}
0 & 0 & g
\end{array}\right]^{T}
$$

Assumption 5: Gravity is constant over the airframe.

Since linear elastic theory was assumed (Assumption 3) the generalized stiffness matrix $\mathbf{K}_{E E}$ does not depend on the structural deformation and the boundary conditions remain constant during deformation. With the vector $\mathbf{R}_{i}$ (8a) (defining the location of the mass element) the potential energy (21) may be expanded to

$$
\begin{aligned}
E_{p o t} & =-\sum_{i}\left(\mathbf{R}_{0}+\overline{\mathbf{r}}_{i}+\overline{\mathbf{d}}_{i}\right)^{T} \mathbf{T}_{e b}^{T} \mathbf{g}_{e} m_{i}+\frac{1}{2} \eta_{E}^{T} \mathbf{K}_{E E} \eta_{E} \\
& =-m \mathbf{R}_{0 e}^{T} \mathbf{g}_{e}+\frac{1}{2} \eta_{E}^{T} \mathbf{K}_{E E} \eta_{E}
\end{aligned}
$$

since $\sum_{i}=\left(\overline{\mathbf{r}}_{i}+\overline{\mathbf{d}}_{i}\right) m_{i}=0$, due to location of the body frame in the momentary center of gravity. 


\section{Virtual Work of Nonconservative Forces}

The Lagrange's equations require the formulation of the nonconservative external forces. Conservative external forces where already accounted for in the formulation of the potential energy. The remaining nonconservative external forces and moments are the aerodynamic and thrust forces. These will be written as load vector $\mathbf{P}_{g}$ collecting the local forces and moments at each grid point:

$$
\mathbf{P}_{g}=\left[\ldots, \mathbf{F}_{i}^{T}, \mathbf{M}_{i}^{T}, \ldots\right]^{T}
$$

The virtual work of the nonconservative forces $\mathbf{F}_{i}$ and moments $\mathbf{M}_{i}$ applied at the grid points i is then given by: ${ }^{17}$

$$
\delta W_{n c o}=\sum_{i}\left(\delta \mathbf{R}_{i}\right)^{T} \mathbf{F}_{i}+\left(\delta \alpha+\delta \varphi_{i}\right)^{T} \mathbf{M}_{i}
$$

where $\delta \alpha$ is the vector of virtual angular displacements of the body frame. The virtual displacement of the grid point i may be written as follows: ${ }^{18}$

$$
\delta \mathbf{R}_{i}=\delta \mathbf{R}_{0}+s k(\delta \alpha) \mathbf{r}_{i}+\delta \mathbf{d}_{i}
$$

Inserting (26) in (25) and applying the modal approach (18) yields:

$$
\begin{aligned}
\delta W_{\text {nco }} & =\sum_{i}\left(\delta \mathbf{R}_{0}+s k(\delta \alpha) \mathbf{r}_{i}+\delta \mathbf{d}_{i}\right)^{T} \mathbf{F}_{i}+\left(\delta \alpha+\delta \varphi_{i}\right)^{T} \mathbf{M}_{i} \\
& =\left[\begin{array}{ll}
\delta \mathbf{R}_{0}^{T} & \delta \alpha^{T}
\end{array}\right] \sum_{i} \underbrace{\left[\begin{array}{cc}
\mathbf{I} & \mathbf{0} \\
s k\left(\mathbf{r}_{i}\right) & \mathbf{I}
\end{array}\right]}_{\mathbf{\Phi}_{g_{i} R}^{T}}\left[\begin{array}{c}
\mathbf{F}_{i} \\
\mathbf{M}_{i}
\end{array}\right]+\delta \eta_{E}^{T} \sum_{i} \boldsymbol{\Phi}_{g_{i} E}^{T}\left[\begin{array}{c}
\mathbf{F}_{i} \\
\mathbf{M}_{i}
\end{array}\right]
\end{aligned}
$$

where the matrix of rigid body modes $\boldsymbol{\Phi}_{g R}$ represents unit translations and rotations along the aircraft body axis w.r.t the center of gravity. The virtual can be written in its final form, using (5):

$$
\begin{aligned}
\delta W_{n c o} & =\left[\begin{array}{ll}
\delta \mathbf{R}_{0}^{T} & \delta \alpha^{T}
\end{array}\right] \mathbf{\Phi}_{g_{R}}^{T} \mathbf{P}_{g}+\delta \eta_{E}^{T} \boldsymbol{\Phi}_{g_{E}}^{T} \mathbf{P}_{g} \\
& =\left[\begin{array}{ll}
\delta \mathbf{R}_{0 e}^{T} \mathbf{T}_{e b} & \delta \mathbf{\Theta}^{T} \mathbf{D}^{T}
\end{array}\right] \mathbf{\Phi}_{g_{R}}^{T} \mathbf{P}_{g}+\delta \eta_{E}^{T} \mathbf{\Phi}_{g_{E}}^{T} \mathbf{P}_{g}
\end{aligned}
$$

and may subsequently be used in Lagrange's equations.

\section{Derivation of the Equations of Motion}

In this section the equations of motion will be derived by applying Lagrange's equations (1). The required terms are given in:

Kinetic Energy: Eq. (20)

Potential Energy: Eq. (23)

Virtual Work: Eq. (28)

Lagrange's equations (1) consist of three vector equations, the force equation (1a), the moment equation (1b) and the elastic equation (1c). These will be successively derived in the following sections.

\section{Force Equation}

First the force equation (1a) is considered. Differentiation of the Lagrange Variable and the virtual work (28) yields

$$
\begin{aligned}
\frac{\partial \mathcal{L}}{\partial \mathbf{V}_{b}} & =\mathbf{V}_{b} m \\
\frac{\partial}{\partial t}\left\{\frac{\partial \mathcal{L}}{\partial \mathbf{V}_{b}}\right\} & =\stackrel{\circ}{\mathbf{V}}_{b} m \\
\mathbf{T}_{b e} \frac{\partial \mathcal{L}}{\partial \mathbf{R}_{0 e}} & =m \mathbf{T}_{b e} \mathbf{g}_{e} \\
\mathbf{T}_{b e} \mathbf{Q}_{t} & =\mathbf{T}_{b e} \frac{\partial\left(\delta W_{n c o}\right)}{\partial \mathbf{R}_{0 e}}=\left(\boldsymbol{\Phi}_{g_{R}}^{T}\right)_{t} \mathbf{P}_{g}
\end{aligned}
$$

$$
7 \text { of } 21
$$


Hence, the equation of motion for the translational degrees of freedom is

$$
m\left[\stackrel{\circ}{\mathbf{V}}_{b}+\boldsymbol{\Omega}_{b} \times \mathbf{V}_{b}-\mathbf{T}_{b e} \mathbf{g}_{e}\right]=\left(\boldsymbol{\Phi}_{g_{R}}^{T}\right)_{t} \mathbf{P}_{g}
$$

where the right hand side of the equation $\left(\boldsymbol{\Phi}_{g_{R}}^{T}\right)_{t} \mathbf{P}_{g}$ represents the sum of all nonconservative external forces.

\section{Moment Equation}

Next the moment equation (1b) is developed. The derivatives of the Lagrange Variable and the virtual work are as follows:

$$
\begin{aligned}
\frac{\partial \mathcal{L}}{\partial \boldsymbol{\Omega}_{b}} & =\mathbf{J} \boldsymbol{\Omega}_{b}+\sum_{i}\left(\overline{\mathbf{d}}_{i} \times \stackrel{\circ}{\mathbf{d}}_{i}\right) m_{i}+\sum_{i} \mathbf{J}_{i} \stackrel{\circ}{\varphi}_{i} \\
\frac{\partial}{\partial t}\left\{\frac{\partial \mathcal{L}}{\partial \boldsymbol{\Omega}_{b}}\right\} & =\mathbf{J} \stackrel{\circ}{\boldsymbol{\Omega}}_{b}+\stackrel{\circ}{\mathbf{J}} \boldsymbol{\Omega}_{b}+\sum_{i}\left(\overline{\mathbf{d}}_{i} \times \stackrel{\circ}{\mathbf{d}}_{i}\right) m_{i}+\sum_{i} \mathbf{J}_{i} \stackrel{\circ}{\varphi}_{i} \\
\frac{\partial \mathcal{L}}{\partial \boldsymbol{\Theta}} & =\mathbf{0} \\
\left(\mathbf{D}^{T}\right)^{-1} \mathbf{Q}_{r} & =\left(\mathbf{D}^{T}\right)^{-1} \frac{\partial\left(\delta W_{n c o}\right)}{\partial \boldsymbol{\Theta}}=\left(\boldsymbol{\Phi}_{g_{R}}^{T}\right)_{r} \mathbf{P}_{g}
\end{aligned}
$$

where the right hand side of the equation $\left(\boldsymbol{\Phi}_{g_{R}}^{T}\right)_{r} \mathbf{P}_{g}$ is the resulting moment of the nonconservative external forces w.r.t. the c.g.. The equation of motion for the rotational degrees of freedom then is

$$
\mathbf{J} \stackrel{\circ}{\boldsymbol{\Omega}}_{b}+\boldsymbol{\Omega}_{b} \times \mathbf{J} \boldsymbol{\Omega}_{b}+\stackrel{\circ}{\mathbf{J}} \boldsymbol{\Omega}_{b}+\stackrel{\circ}{\mathbf{h}}+\boldsymbol{\Omega}_{b} \times \mathbf{h}=\left(\Phi_{g_{R}}^{T}\right)_{r} \mathbf{P}_{g}
$$

with

$$
\begin{gathered}
\mathbf{h}=\sum_{i}\left(\overline{\mathbf{d}}_{i} \times \stackrel{\circ}{\mathbf{d}}_{i}\right) m_{i}+\sum_{i} \mathbf{J}_{i} \stackrel{\circ}{\varphi}_{i} \\
\stackrel{\circ}{\mathbf{h}}=\sum_{i}\left(\overline{\mathbf{d}}_{i} \times \stackrel{\circ}{\mathbf{d}}_{i}\right) m_{i}+\sum_{i} \mathbf{J}_{i} \stackrel{\circ}{\varphi}_{i}
\end{gathered}
$$

and the inertia tensor $\mathbf{J}$ defined in (16).

The previous moment equation includes the inertial coupling terms $\mathbf{h}, \stackrel{\circ}{\mathbf{h}}$ and $\mathbf{J}, \stackrel{\circ}{\mathbf{J}}$. Note that one can obtain the formulation published by Buttrill ${ }^{4}$ from the present moment equation (32), if mass offsets and elastic rotational degrees of freedoms are neglected. The above moment equation has one drawback for simulation. The sums over all grid points in the terms $\mathbf{h}, \stackrel{\circ}{\mathbf{h}}$ and $\mathbf{J}, \stackrel{\circ}{\mathbf{J}}$ have to be recalculated at every time step. This can slow down the simulation rate significantly. Therefore it is desirable to eliminate the grid point sums in (32) by including the modal approach in the inertial coupling terms. A generalized formulation of the inertial coupling terms is also required for the derivation of the elastic equation, since the Lagrange equation includes derivatives by the generalized elastic coordinates. The generalization process is (Appendix VII) yields the generalized expressions:

$$
\begin{aligned}
& \text { Eq.(75) : } \quad \mathbf{J}=\sum_{i=} \mathbf{J}_{g, i}-A 1-\left\langle\eta_{E}^{T} \widetilde{B}_{j k} \eta_{E}\right\rangle-\left\langle\eta_{E}^{T} \widetilde{C}_{j k}+\widetilde{D}_{j k} \eta_{E}\right\rangle
\end{aligned}
$$

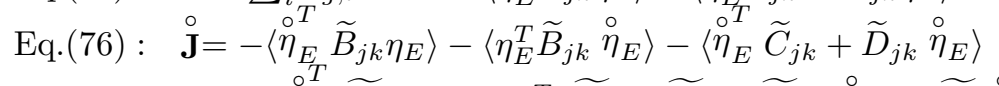

$$
\begin{aligned}
& \text { Eq.(82): } \quad \mathbf{h}=-\left\langle\stackrel{\circ}{\eta}_{E} \widetilde{h 2}_{j} \eta_{E}\right\rangle+\left\langle\eta_{E}^{T}\left(\widetilde{h 1}_{j}+\widetilde{h 2}{ }_{j}+\widetilde{h 4}{ }_{j}\right) \stackrel{\circ}{\eta}_{E}\right\rangle+\widetilde{h 5} \stackrel{\circ}{\eta}_{E} \\
& \text { Eq. (83) : } \quad \stackrel{\circ}{\mathbf{h}}=\left\langle\stackrel{\circ}{\eta}_{E}\left(\widetilde{h 1}_{j}+\widetilde{h 4}_{j}\right) \stackrel{\circ}{\eta}_{E}\right\rangle-\left\langle\stackrel{\circ}{\eta}_{E} \widetilde{h 2}_{j} \eta_{E}\right\rangle+\left\langle\eta_{E}^{T}\left(\widetilde{h 1}_{j}+\widetilde{h 2_{j}}+\widetilde{h 4}_{j}\right) \stackrel{\circ}{\eta}_{E}\right\rangle
\end{aligned}
$$

see (62) for definition of the $\langle\ldots\rangle$ notation. 


\section{Elastic Equation}

The third equation in Lagrange's equations is the elastic equation (1c). The following derivatives are needed:

$$
\begin{aligned}
\frac{\partial}{\partial t}\left\{\frac{\partial \mathcal{L}}{\partial{\stackrel{\circ}{\eta_{E}}}}\right\} & =\frac{\partial}{\partial t}\left\{\frac{\partial T_{k i n}}{\partial{\stackrel{\circ}{\eta_{E}}}}-\frac{\partial T_{p o t}}{\partial \stackrel{\circ}{\eta}_{E}}\right\} \\
\frac{\partial \mathcal{L}}{\partial \eta_{E}} & =\frac{\partial T_{k i n}}{\partial \eta_{E}}-\frac{\partial T_{p o t}}{\partial \eta_{E}} \\
\mathbf{Q}_{E} & =\frac{\partial\left(\delta W_{n c o}\right)}{\partial \eta_{E}}=\boldsymbol{\Phi}_{g_{E}}^{T} \mathbf{P}_{g}
\end{aligned}
$$

With the expression for the kinetic energy $(20)$ and the $\mathbf{h}$-term $(82)$ the derivative $\frac{\partial T_{k i n}}{\partial \eta_{E}}$ becomes

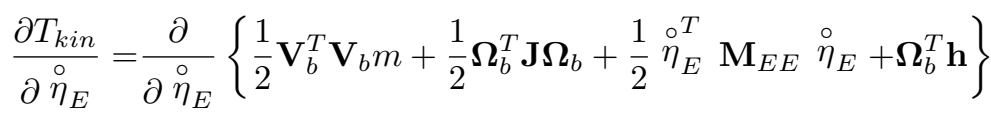

$$
\begin{aligned}
& =\mathbf{M}_{E E} \stackrel{\circ}{\eta}_{E}+\frac{\partial}{\partial \stackrel{\circ}{\eta}_{E}}\left\{\boldsymbol{\Omega}_{b}^{T} \mathbf{h}\right\}
\end{aligned}
$$

where the $\mathbf{h}$-term (82) may be written in the following form:

$$
\mathbf{h}=\sum_{j=1}^{3}\left(-\stackrel{\circ}{\eta}_{E}^{T} \widetilde{h 2}_{j} \eta_{E}+\eta_{E}^{T}\left(\widetilde{h 1}_{j}+\widetilde{h 2}_{j}+\widetilde{h 4}_{j}\right) \stackrel{\circ}{\eta}_{E}\right) \mathbf{e}_{j}+\widetilde{h 5} \stackrel{\circ}{\eta}_{E}
$$

Then the derivative (35) can be expressed as follows:

$$
\frac{\partial T_{k i n}}{\partial \stackrel{\circ}{\eta}_{E}}=\mathbf{M}_{E E} \stackrel{\circ}{\eta}_{E}+\frac{\partial}{\partial \stackrel{\circ}{\eta}_{E}}\left\{\sum_{j=1}^{3}\left(-\stackrel{\circ}{\eta}_{E} \widetilde{h 2_{j}}{ }_{\eta_{E}}+\eta_{E}^{T}\left(\widetilde{h 1}_{j}+\widetilde{h 2}_{j}+\widetilde{h 4}_{j}\right) \stackrel{\circ}{\eta}_{E}\right) \boldsymbol{\Omega}_{b}^{T} \mathbf{e}_{j}+\boldsymbol{\Omega}_{b}^{T} \widetilde{h 5} \stackrel{\circ}{\eta}_{E}\right\}
$$

applying the differentiation $\frac{\partial}{\partial \stackrel{\circ}{\eta}_{E}}$ in $(37)$ yields:

$$
\frac{\partial T_{k i n}}{\partial \stackrel{\circ}{\eta}_{E}}=\mathbf{M}_{E E}{\stackrel{\circ}{\eta_{E}}}+\sum_{j=1}^{3} \underbrace{\left(\widetilde{h 1}_{j}^{T}-\widetilde{h 2}+\widetilde{h 2}_{j}^{T}+\widetilde{h 4}_{j}^{T}\right)}_{\widetilde{h}_{j}} \eta_{E} \boldsymbol{\Omega}_{b}^{T} \mathbf{e}_{j}+\widetilde{h 5}^{T} \boldsymbol{\Omega}_{b}
$$

where the term $\widetilde{h}_{j}$ is defined to simplify the expression. Next the potential energy is considered. It simply becomes $\frac{\partial T_{p o t}}{\partial \grave{\eta}_{E}}=0$. The additional derivative $\frac{\partial}{\partial t}$ applied on (38) yields:

$$
\frac{\partial}{\partial t}\left\{\frac{\partial \mathcal{L}}{\partial \stackrel{\circ}{\eta}_{E}}\right\}=\mathbf{M}_{E E} \stackrel{\circ}{\eta^{\circ}} E+\sum_{j=1}^{3} \widetilde{h}_{j}\left(\stackrel{\circ}{\eta}_{E} \mathbf{\Omega}_{b}^{T}+\eta_{E}{\stackrel{\circ}{\boldsymbol{\Omega}_{b}}}^{T}\right) \mathbf{e}_{j}+\widetilde{h 5}^{T} \stackrel{\circ}{\Omega_{b}}
$$

The derivative $\frac{\partial T_{k i n}}{\partial \eta_{E}}$ is considered next

$$
\begin{aligned}
\frac{\partial T_{k i n}}{\partial \eta_{E}} & =\frac{\partial}{\partial \eta_{E}}\left\{\frac{1}{2} \mathbf{V}_{b}^{T} \mathbf{V}_{b} m+\frac{1}{2} \boldsymbol{\Omega}_{b}^{T} \mathbf{J} \boldsymbol{\Omega}_{b}+\frac{1}{2} \stackrel{\circ}{\eta_{E}^{T}} \mathbf{M}_{E E} \stackrel{\circ}{\eta}_{E}+\boldsymbol{\Omega}_{b}^{T} \mathbf{h}\right\} \\
& =\frac{1}{2} \frac{\partial}{\partial \eta_{E}}\left\{\boldsymbol{\Omega}_{b}^{T} \mathbf{J} \boldsymbol{\Omega}_{b}\right\}+\frac{\partial}{\partial \eta_{E}}\left\{\boldsymbol{\Omega}_{b}^{T} \mathbf{h}\right\}
\end{aligned}
$$

where the first term can be written as follows

$$
\begin{aligned}
\frac{\partial}{\partial \eta_{E}}\left\{\boldsymbol{\Omega}_{b}^{T} \mathbf{J} \boldsymbol{\Omega}_{b}\right\} & =-\frac{\partial}{\partial \eta_{E}} \sum_{j=1}^{3} \sum_{k=1}^{3}\left(\eta_{E}^{T} \widetilde{B}_{j k} \eta_{E}+\eta_{E}^{T} \widetilde{C}_{j k}+\widetilde{D}_{j k} \eta_{E}\right)\left(\boldsymbol{\Omega}_{b}^{T} \mathbf{e}_{j} \mathbf{e}_{k}^{T} \mathbf{\Omega}_{b}\right) \\
& =-\sum_{j=1}^{3} \sum_{k=1}^{3}\left(\left(\widetilde{B}_{j k}+\widetilde{B}_{j k}^{T}\right) \eta_{E}+\widetilde{C}_{j k}+\widetilde{D}_{j k}^{T}\right)\left(\mathbf{\Omega}_{b}^{T} \mathbf{e}_{j} \mathbf{e}_{k}^{T} \boldsymbol{\Omega}_{b}\right)
\end{aligned}
$$


and the second term is given by:

$$
\begin{aligned}
\frac{\partial}{\partial \eta_{E}}\left\{\boldsymbol{\Omega}_{b}^{T} \mathbf{h}\right\} & =\frac{\partial}{\partial \eta_{E}}\left\{\sum_{j=1}^{3}\left(-\stackrel{\circ}{\eta}_{E}^{T} \widetilde{h 2}_{j} \eta_{E}+\eta_{E}^{T}\left(\widetilde{h 1}_{j}+\widetilde{h 2}_{j}+\widetilde{h 4}_{j}\right) \stackrel{\circ}{\eta}_{E}\right) \boldsymbol{\Omega}_{b}^{T} \mathbf{e}_{j}+\boldsymbol{\Omega}_{b}^{T} \widetilde{h 5} \stackrel{\circ}{\eta}_{E}\right\} \\
& =\sum_{j=1}^{3} \underbrace{\left(\widetilde{h 1}_{j}-\widetilde{h 2}_{j}^{T}+\widetilde{h 2}_{j}+\widetilde{h 4}_{j}\right)}_{\widetilde{h}_{j}^{T}}{\stackrel{\circ}{\eta}}_{E} \boldsymbol{\Omega}_{b}^{T} \mathbf{e}_{j}
\end{aligned}
$$

The derivative $\frac{\partial T_{p o t}}{\partial \eta_{E}}$ is as follows: $\frac{\partial T_{p o t}}{\partial \eta_{E}}=\mathbf{K}_{E E} \eta_{E}$. Incorporating the preceding derivatives into the elastic equation of motion yields:

$$
\begin{aligned}
\mathbf{M}_{E E} \stackrel{\circ}{\eta}_{E}+\mathbf{K}_{E E} \eta_{E} & +\sum_{j=1}^{3} \widetilde{h}_{j}\left(\stackrel{\circ}{\eta}_{E} \boldsymbol{\Omega}_{b}^{T}+\eta_{E} \stackrel{\circ}{\boldsymbol{\Omega}_{b}}\right) \mathbf{e}_{j}+\widetilde{h 5}^{T} \stackrel{\circ}{\boldsymbol{\Omega}_{b}} \\
& +\frac{1}{2} \sum_{j=1}^{3} \sum_{k=1}^{3}\left(\left(\widetilde{B}_{j k}+\widetilde{B}_{j k}^{T}\right) \eta_{E}+\widetilde{C}_{j k}+\widetilde{D}_{j k}^{T}\right) \boldsymbol{\Omega}_{b}^{T} \mathbf{e}_{j} \mathbf{e}_{k}^{T} \boldsymbol{\Omega}_{b}-\sum_{j=1}^{3} \widetilde{h}_{j}^{T} \stackrel{\circ}{\eta}_{E} \boldsymbol{\Omega}_{b}^{T} \mathbf{e}_{j}=Q
\end{aligned}
$$

Reordering of the terms and inclusion of structural damping via the diagonal modal damping matrix: ${ }^{2}$

$$
\mathbf{B}_{E E}=2 \operatorname{diag}\left(\zeta_{i}\right)\left(\mathbf{M}_{E E} \mathbf{K}_{E E}\right)^{1 / 2}
$$

yields the final form of the elastic equation with generalized coupling terms:

$$
\begin{aligned}
\mathbf{M}_{E E} \stackrel{\circ}{\eta}_{E}+\mathbf{B}_{E E} \stackrel{\circ}{\eta}_{E}+\mathbf{K}_{E E} \eta_{E}+ & \underbrace{\left(\sum_{j=1}^{3} \widetilde{h}_{j} \eta_{E} \mathbf{e}_{j}^{T}+\widetilde{h 5}^{T}\right) \stackrel{\circ}{\boldsymbol{\Omega}}_{b}}_{\text {due to angular acc. of the body frame }}+\underbrace{2 \sum_{j=1}^{3} \widetilde{h}_{j} \stackrel{\circ}{\eta}_{E} \mathbf{e}_{j}^{T} \boldsymbol{\Omega}_{b}}_{\text {Coriolis term }} \\
& +\underbrace{\frac{1}{2} \sum_{j=1}^{3} \sum_{k=1}^{3}\left(\left(\widetilde{B}_{j k}+\widetilde{B}_{j k}^{T}\right) \eta_{E}+\widetilde{C}_{j k}+\widetilde{D}_{j k}^{T}\right) \boldsymbol{\Omega}_{b}^{T} \mathbf{e}_{j} \mathbf{e}_{k}^{T} \boldsymbol{\Omega}_{b}}_{\text {centrifugal loading on the elastic modes }}=\boldsymbol{\Phi}_{g_{E}}^{T} \mathbf{P}_{g}
\end{aligned}
$$




\section{Equations of Structural Loads}

To derive the loads equation for a free flying flexible aircraft with inertially coupled equations of motion one has to start with the principle of momentum. The principle of momentum is given by: ${ }^{13}$

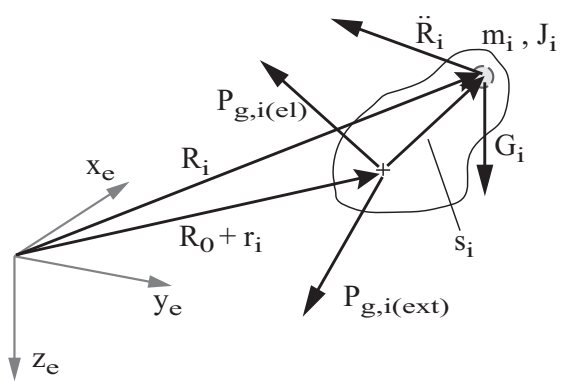

Figure 2. External and elastic forces grid point i

$$
\frac{d}{d t}\left[\begin{array}{l}
\mathbf{H}_{t, i} \\
\mathbf{H}_{r, i}
\end{array}\right]=\left[\begin{array}{c}
\mathbf{F}_{i} \\
\mathbf{M}_{i}
\end{array}\right]
$$

where $\mathbf{H}_{t, i}, \mathbf{H}_{r, i}$ denotes the resulting translational and rotational momentum vector and $\mathbf{F}_{i}, \mathbf{M}_{i}$ at the location of the mass i. The linear and angular momentum of the lumped mass i (left hand side of (46) ) may be written as follows: ${ }^{13}$

$$
\left[\begin{array}{l}
\mathbf{H}_{t, i} \\
\mathbf{H}_{r, i}
\end{array}\right]=\left[\begin{array}{cc}
m_{i} \mathbf{I} & \mathbf{0} \\
\mathbf{0} & \mathbf{J}_{i}
\end{array}\right]\left[\begin{array}{l}
\dot{\mathbf{R}}_{i} \\
\boldsymbol{\Omega}_{i}
\end{array}\right]
$$

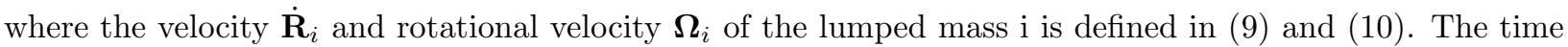
derivative of the momentum (47) is given by:

$$
\frac{d}{d t}\left[\begin{array}{l}
\mathbf{H}_{t, i} \\
\mathbf{H}_{r, i}
\end{array}\right]=\left[\begin{array}{c}
m_{i} \ddot{\mathbf{R}}_{i} \\
\mathbf{J}_{i}\left(\stackrel{\circ}{\boldsymbol{\Omega}}_{b}+\stackrel{\circ}{\varphi}_{i}\right)+s k\left(\boldsymbol{\Omega}_{b}\right) \mathbf{J}_{i}\left(\boldsymbol{\Omega}_{b}+\stackrel{\circ}{\varphi}_{i}\right)
\end{array}\right]
$$

The resulting force and moment at the grid point $\mathbf{F}_{i}, \mathbf{M}_{i}$ (right hand side of (46)) is expanded to elastic forces, gravity forces, and other external forces:

$$
\left[\begin{array}{c}
\mathbf{F}_{i} \\
\mathbf{M}_{i}
\end{array}\right]=\left[\begin{array}{c}
\mathbf{P}_{g t, i}^{e x t} \\
\mathbf{P}_{g r, i}^{e x t}
\end{array}\right]+\left[\begin{array}{c}
\mathbf{P}_{g, t}^{e l} \\
\mathbf{P}_{g r, i}^{e l}
\end{array}\right]+\left[\begin{array}{c}
\mathbf{0} \\
-s k\left(\mathbf{s}_{i}\right)\left(\mathbf{P}_{g t, i}^{e x t}+\mathbf{P}_{g t, i}^{e l}\right)
\end{array}\right]+\left[\begin{array}{c}
\mathbf{G}_{i} \\
\mathbf{0}
\end{array}\right]
$$

Note that $\mathbf{s}_{i}$ represents the mass offset (Fig. 2). Therefore the term $-s k\left(\mathbf{s}_{i}\right)\left(\mathbf{P}_{g t, i}^{e x t}+\mathbf{P}_{g t, i}^{e l}\right)$ is the moment due to the forces $\mathbf{P}_{g t, i}^{e x t}+\mathbf{P}_{g t, i}^{e l}$ acting at the grid point location w.r.t the location of the mass element. With the external forces (49) and the time derivative of the momentum (48) the principle of momentum (46) can be written in the following form:

$$
\left[\begin{array}{c}
m_{i} \ddot{\mathbf{R}}_{i} \\
\mathbf{J}_{i}\left(\stackrel{\circ}{\boldsymbol{\Omega}}_{b}+\stackrel{\circ}{\varphi}_{i}\right)+s k\left(\boldsymbol{\Omega}_{b}\right) \mathbf{J}_{i}\left(\boldsymbol{\Omega}_{b}+\stackrel{\circ}{\varphi}_{i}\right)
\end{array}\right]=\left[\begin{array}{c}
\mathbf{P}_{g t, i}^{e x t} \\
\mathbf{P}_{g r, i}^{e x t}
\end{array}\right]+\left[\begin{array}{c}
\mathbf{P}_{g t, i}^{e l} \\
\mathbf{P}_{g r, i}^{e l}
\end{array}\right]+\left[\begin{array}{c}
\mathbf{0} \\
-s k\left(\mathbf{s}_{i}\right)\left(\mathbf{P}_{g t, i}^{e x t}+\mathbf{P}_{g t, i}^{e l}\right)
\end{array}\right]+\left[\begin{array}{c}
\mathbf{G}_{i} \\
\mathbf{0}
\end{array}\right]
$$

Further development of the above equations yields:

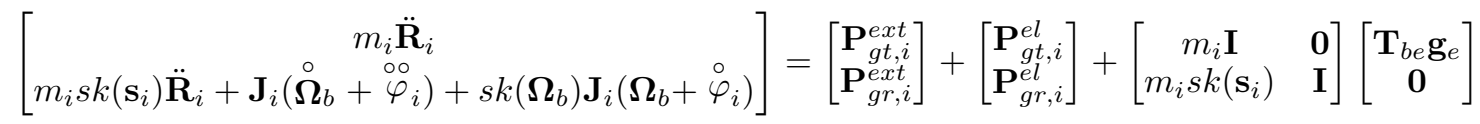

where the gravitational force on the mass element $\mathrm{i}$ is expressed by (22) as $\mathbf{G}_{i}=m_{i} \mathbf{T}_{b e} \mathbf{g}_{e}$. Expression (51) is now solved for the resulting elastic forces acting from the grid point $i$ on the neighbored grid points, hence: $\mathbf{L}_{i}=-\left[\begin{array}{c}\mathbf{P}_{g t, i}^{e l} \\ \mathbf{P}_{g r, i}^{e l}\end{array}\right]$, the preliminary form of the Force Summation Method then becomes:

$$
\mathbf{L}_{g, i}^{F S M}=\left[\begin{array}{c}
\mathbf{P}_{g t, i}^{e x t} \\
\mathbf{P}_{g r, i}^{e x t}
\end{array}\right]-\left[\begin{array}{cc}
m_{i} \mathbf{I} & \mathbf{0} \\
m_{i} s k\left(\mathbf{s}_{i}\right) & \mathbf{J}_{i}
\end{array}\right]\left[\begin{array}{c}
\ddot{\mathbf{R}}_{i}-\mathbf{T}_{b e} \mathbf{g}_{e} \\
\stackrel{\circ}{\boldsymbol{\Omega}}_{b}+\stackrel{\circ}{\varphi}_{i}
\end{array}\right]-\left[\begin{array}{c}
\mathbf{0} \\
s k\left(\boldsymbol{\Omega}_{b}\right) \mathbf{J}_{i}
\end{array}\right]\left(\boldsymbol{\Omega}_{b}+\stackrel{\circ}{\varphi}_{i}\right)
$$


where translational inertial acceleration of the local mass element $m_{i}$ is given by differentiation of (9)

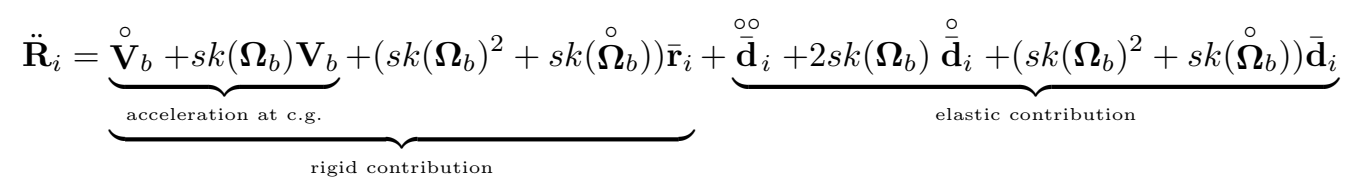

Elastic displacements, velocities and accelerations are now expressed using the modal approach (18):

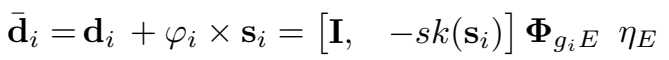

$$
\begin{aligned}
& \stackrel{\circ}{\mathbf{d}}_{i}=\stackrel{\circ}{\mathbf{d}}_{i}+\stackrel{\circ}{\varphi}_{i} \times \mathbf{s}_{i}=\left[\mathbf{I}, \quad-s k\left(\mathbf{s}_{i}\right)\right] \mathbf{\Phi}_{g_{i} E}{\stackrel{\circ}{\eta}} \\
& \stackrel{\circ}{\mathbf{d}}_{i}=\stackrel{\circ}{\mathbf{d}} i+\stackrel{\circ}{\varphi}_{i} \times \mathbf{s}_{i}=\left[\mathbf{I}, \quad-s k\left(\mathbf{s}_{i}\right)\right] \mathbf{\Phi}_{g_{i} E} \stackrel{\circ}{\eta}_{E}
\end{aligned}
$$

Introduction of (53) and (54) in (52) and using

$$
\underbrace{\left[\begin{array}{cc}
m_{i} \mathbf{I} & -m_{i} s k\left(\mathbf{s}_{i}\right) \\
m_{i} s k\left(\mathbf{s}_{i}\right) & \mathbf{J}_{g, i}
\end{array}\right]}_{\mathbf{M}_{g g, i}} \underbrace{\left[\begin{array}{cc}
\mathbf{I} & -s k\left(\mathbf{r}_{i}\right) \\
\mathbf{0} & \mathbf{I}
\end{array}\right]}_{\mathbf{\Phi}_{g R, i}}=\underbrace{\left[\begin{array}{cc}
m_{i} \mathbf{I} & -m_{i} s k\left(\mathbf{r}_{i}+\mathbf{s}_{i}\right) \\
m_{i} s k\left(\mathbf{s}_{i}\right) & \mathbf{J}_{g, i}-m_{i} s k\left(\mathbf{s}_{i}\right) s k\left(\mathbf{r}_{i}\right)
\end{array}\right]}_{\mathbf{M}_{g R, i}}
$$

yields the force summation method:

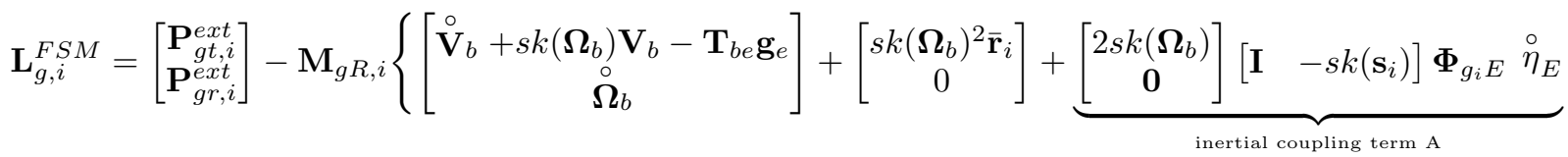

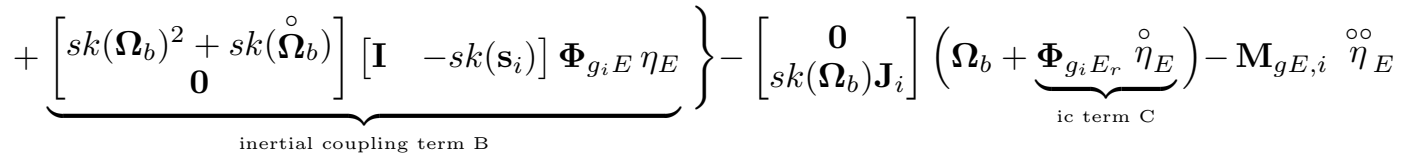

where $\mathbf{V}_{b}, \stackrel{\circ}{\mathbf{V}}_{b}$ is obtained from force equation $(\overline{30}), \boldsymbol{\Omega}_{b}, \stackrel{\circ}{\boldsymbol{\Omega}}_{b}$ from the moment equation (32) and $\eta_{E}, \stackrel{\circ}{\eta}_{E}, \stackrel{\circ}{\eta}_{E}$ from the elastic equation of motion (45).

\section{A. Discussion of the Force Summation Method}

The Force Summation Method (55), derived in the previous section is based on the same assumption as the equations of motion. It may therefore be used for the computation of local forces and moments over the airframe, based on the simulation results of the equations of motion (30), (32) and (45).

The terms denoted by A,B,C in (55) represent the inertial coupling between rigid and elastic motion; already pointed out for the equations of motion. It can be seen that $\mathrm{A}$ and $\mathrm{B}$ represent the coriolis terms and the centrifugal loading. The term $\mathrm{C}$ represents the effect of the variable inertia tensor in the moment equation.

\section{B. Validation of the Force Summation Method}

Elastic forces contained in the equations of motion and the loads equation can not be compared directly. The generalized equation of motion yields elastic forces on the elastic modes, whereas the loads equation yields elastic forces at nodal coordinates. Still both must be consistent. For validation of the loads equation the loads are therefore transformed from physical into modal space by pre multiplication with the elastic mode shapes:

$$
\mathbf{L}_{E}^{F S M}=\boldsymbol{\Phi}_{g_{E}}^{T} \mathbf{L}_{g}^{F S M}
$$

For practical reasons this is done in simulation. A comparison of the generalized load from the loads equation (56) and the elastic forces from the equation of motion (45) is shown in figure 3. Loads computed on basis of the conventional force summation method ( $(55)$ w/o inertial coupling terms) are also depicted. It can be seen that the previously derived loads equations (55) is consistent to the coupled equations of motion formulation. The conventional loads equation is not consistent with the present equations of motion and may not be used if inertial coupling is considered. 

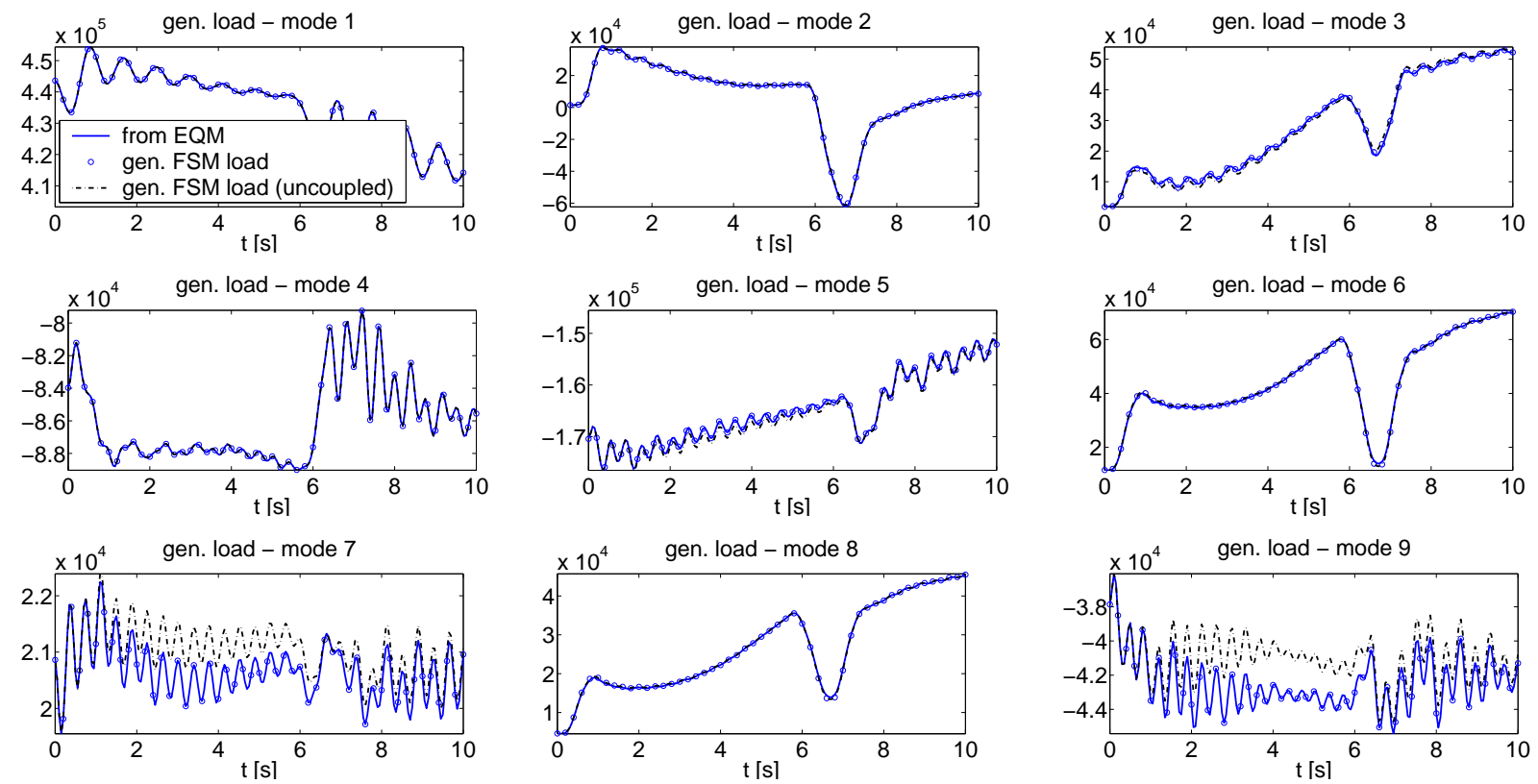

Figure 3. Generalized loads from Equations of Motion and Force Summation Methods

\section{Modelling of External Forces}

External forces are aerodynamic and propulsion force. An aerodynamic module based on the Doublet Lattice theory, ${ }^{1}$ corrected by experimental data, is used for the computation of aerodynamic forces. The

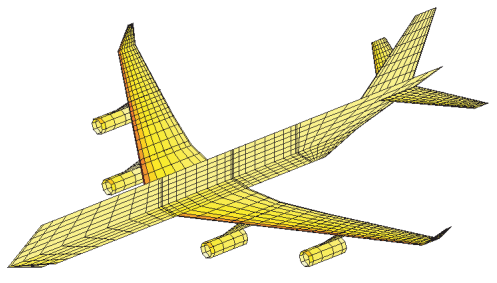

Figure 4. Doubled lattice grid

Double Lattice method is based on linearized aerodynamic potential theory. Lifting surfaces are discretized as small panels, depicted in figure 4.

The correction of the AIC matrix with experimental data is based on an aerodynamic database. The aerodynamic database combines data from CFD calculations, wind tunnel tests and flight test results. The database driven aerodynamic loads can include nonlinearities, e.g. cross coupling terms of angle of attack and side slip angle $\alpha \beta$, or $\beta^{2}$.

The aerodynamic and structural model is interconnected via a spline matrix $\mathbf{T}_{k g}$. The corrected aerodynamic forces can then be written as follows:

$$
\mathbf{P}_{g}^{a e r o}=q_{\infty} \mathbf{T}_{k g}^{T} \mathbf{S}_{k j} \mathbf{Q}_{j j} \mathbf{D}_{j k} \mathbf{T}_{k g}\left[\boldsymbol{\Phi}_{g R}, \mathbf{\Phi}_{g E}\right]\left[\eta_{R}^{T}, \eta_{E}^{T}\right]^{T}
$$

where the AIC matrix depends on Mach number and reduced frequency. For simulation a rational function approximation (RFA) is employed in order to transform the aerodynamic forces from frequency into time domain. ${ }^{22}$ Propulsion forces $\mathbf{P}_{g}^{\text {prop }}$ are accounted for as extern local forces applied at the respective engine nodes. Aerodynamic and propulsion forces are combined to yield the external forces:

$$
\mathbf{P}_{g}^{e x t}=\mathbf{P}_{g}^{\text {aero }}+\mathbf{P}_{g}^{\text {prop }}
$$

$$
13 \text { of } 21
$$




\section{Simulation Results}

A generic large transport aircraft is used for simulation. The simulation is based on the equations of motion (30), (32), (45) and the equation of the external forces (58).

As example case for a dynamic manoeuvre the FAR 25 roll manoeuvre was chosen. The initial condition

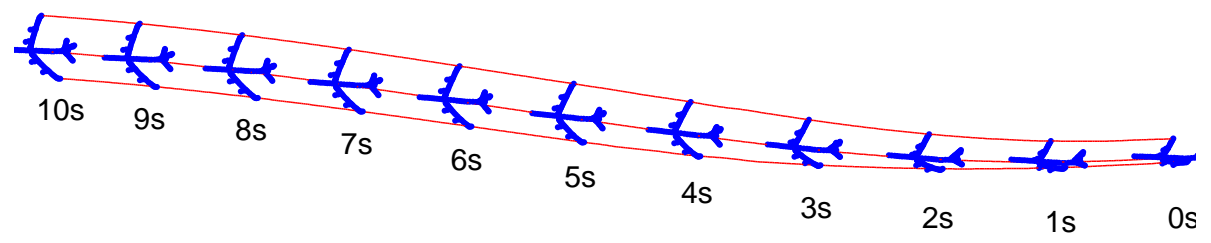

Figure 5. Flight Path - 1.67g example roll manoeuvre

of the roll manoeuvre is a horizontal pull up trimmed with a vertical load factor of $n_{z}=1.67 \mathrm{~g}$. Then roll is initiated via aileron input (Fig. 6) until a high roll rate condition $(\mathrm{t}=3.5 \mathrm{~s})$ and high bank angle is reached. Next opposite aileron deflection is applied initiating a high roll acceleration condition $(\mathrm{t}=3.5-4.5 \mathrm{~s})$. Figure 5 shows the roll maneuver, as seen by an inertial observer. The moment equation (32) accounts for variation
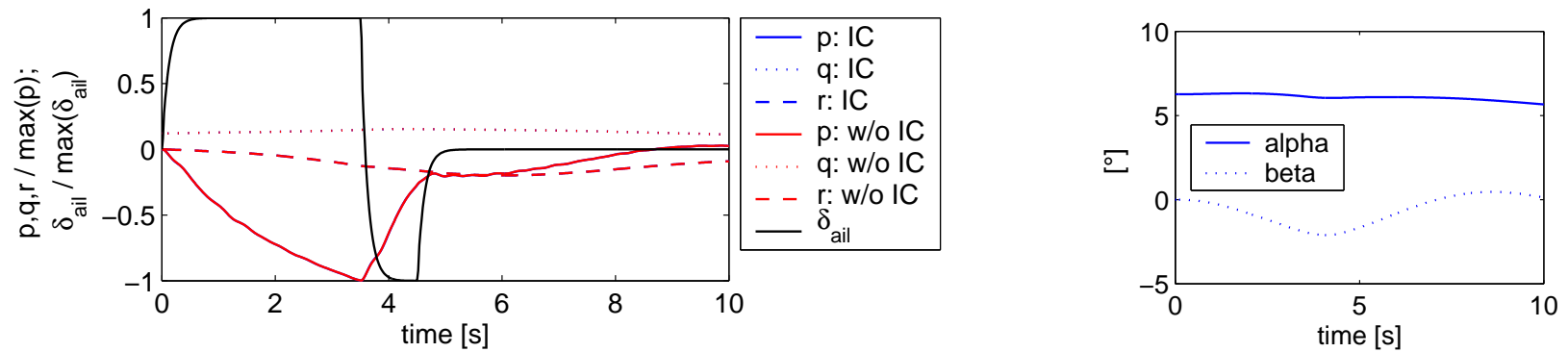

Figure 6. Flight mechanics data for test manoeuvre

of the inertial tensor of the vehicle. Figure 7 depicts the variation of the inertia tensor with time. Especially the elements $J_{x y}$ and $J_{y z}$ noticeably change with elastic deformation.
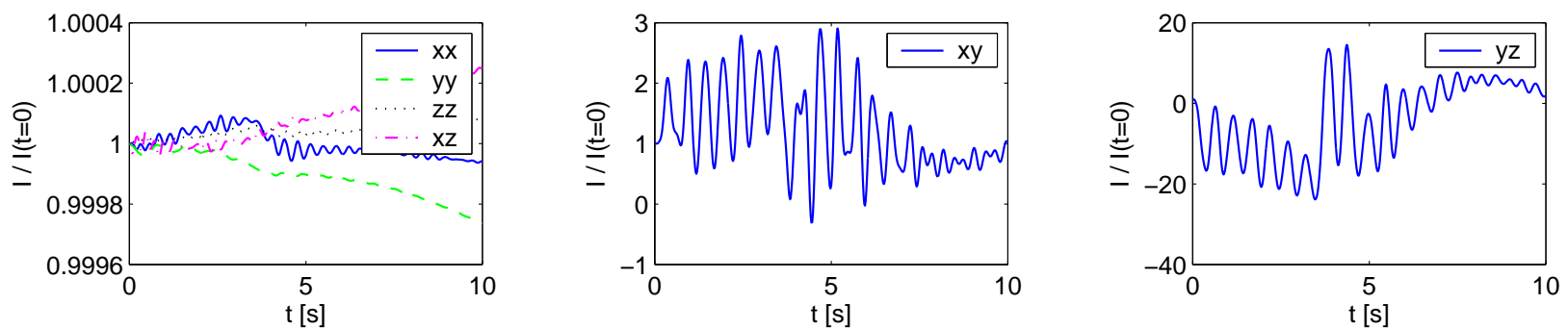

Figure 7. Time variation of the inertia tensor $\mathbf{J}$

\section{A. Loads}

The equation of structural loads $(\underline{55)}$ is used to computed nodal elastic forces over the airframe. Recalling the equations for the kinetic energy (11) it is obvious that inertial coupling has significant influence at nodes where elastic deformation and large local masses are present. For a conventional transport aircraft engines are significantly subjected to inertial coupling effects. The lateral nodal forces at the right outer pylon node (Fig. 8) shows the correlation of roll rate and acceleration with structural loads. Major differences in lateral loads are encountered at the time where maximum roll rate is reached. 


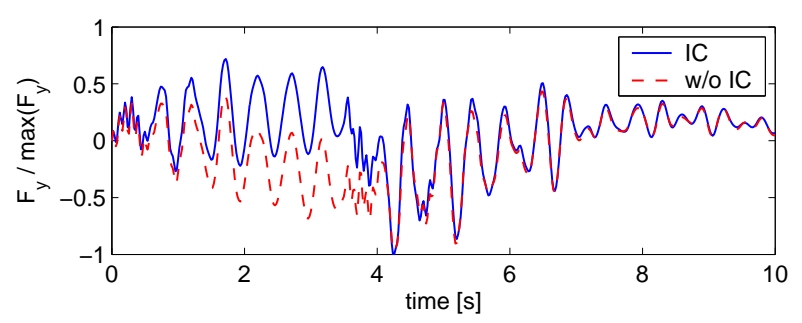

Figure 8. Nodal lateral force at left engine grid

Integrated shear loads will be studied next. A loads envelope for the left wing due to the given manoeuvre is depicted in 9 . The distributed minimum and maximum integrated shear loads are obtained from simulation with inertially uncoupled and coupled equations of motion.
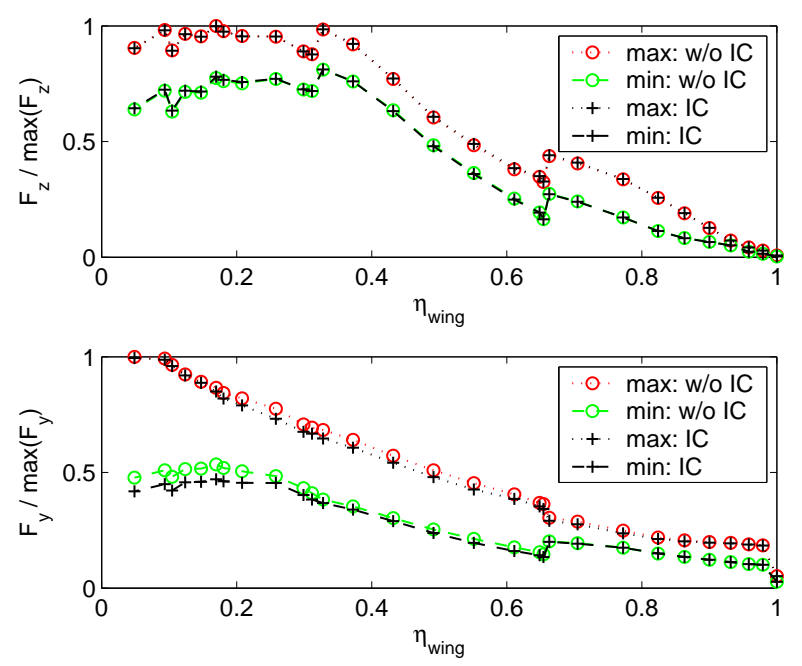

Figure 9. Envelope of test manoeuvre - integrated shear loads

The vertical shear forces $F_{z}$ are not noticeably influenced by inertial coupling effects since aerodynamic forces and gravity forces are the driving forces for shear loads. The situation of different for lateral loads $F_{y}$, figure 9. Aerodynamic components are small and inertial coupling forces, e.g. centrifugal forces are significant in the lateral direction. Especially the outer engine, located at $\eta_{w i n g}=0.65$, causes a difference between coupled and uncoupled simulation.

Figure 10 depicts an overview of the maximum differences for coupled and uncoupled simulation. As already mentioned engines and pylons cause the main differences in lateral forces. Lateral forces also affect the local moments due to mass offset. The high differences in local moments $M_{z}$ are caused by this modelling aspect. The chosen test manoeuvre shows significant influence of inertial coupling on local and integrated loads for:

- hight angular rate / acceleration conditions

- highly flexible structures or structural components

- nodes with large concentrated masses

- force components where external forces are small

\section{Summary and Conclusion}

The equations of motion for an elastic aircraft where derived using Lagrange's equations. The equations are given for a system with discrete masses, rotational degrees of freedom and mass offsets. Therefore available data from FE-models used in loads and aeroelastics can be incorporated directly. 

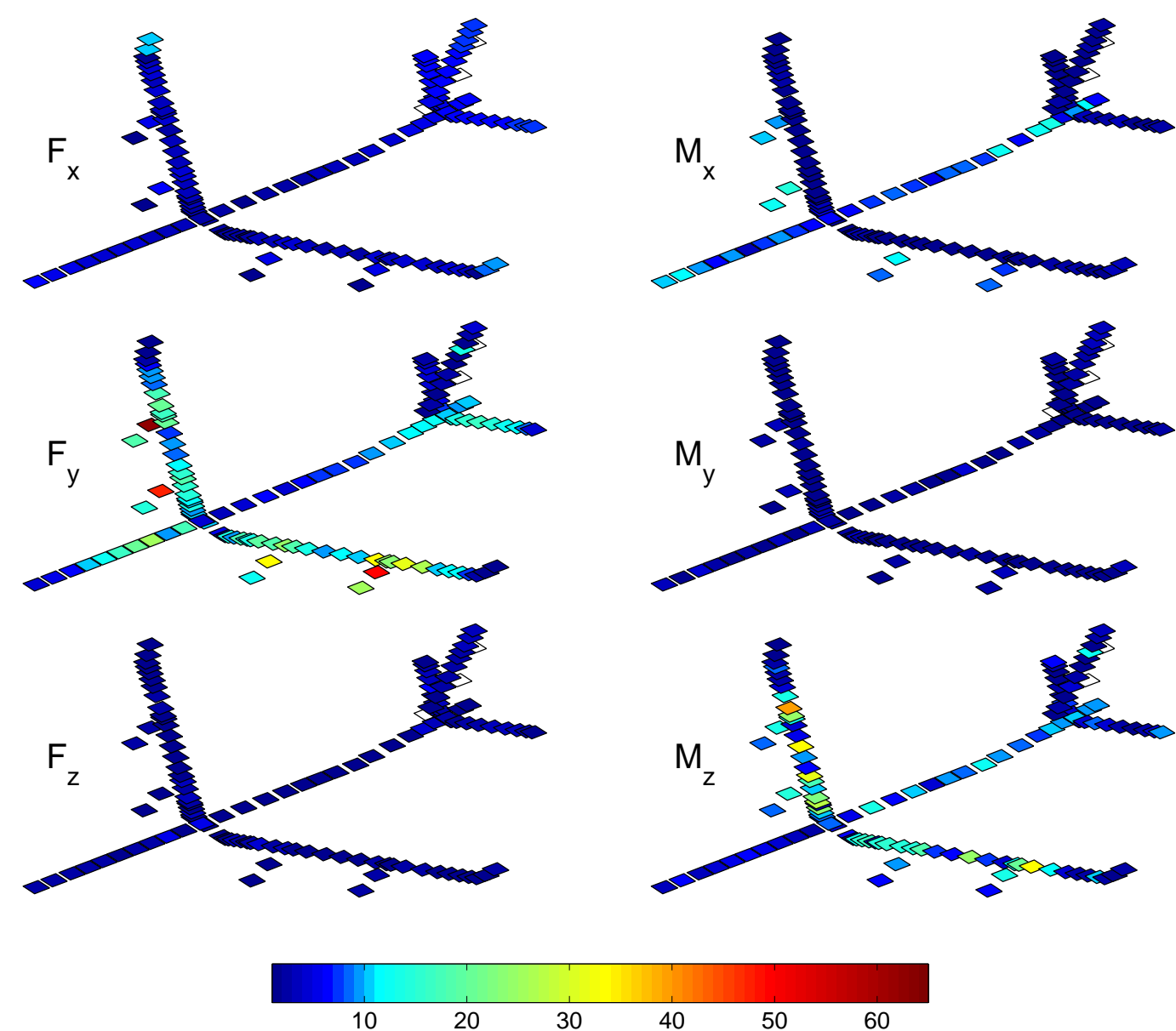

Figure 10. Relative differences [\%] of maximum nodal forces (inertially coupled/uncoupled EOM,FSM) during dynamic simulation

The equations include all inertial coupling terms. The inertia tensor for the deformed aircraft and the additional $h$-term provide coupling of the moment equation with the elastic equation. The forces from angular accelerations of the body frame, Coriolis forces and the centrifugal loading on the elastic modes provide coupling of the elastic equation with the moment equation.

All coupling terms are cast in generalized matrix form for computational efficiency. The modal form is validated by comparison with the physical form. The necessary matrices can be build in preprocessing from available FE data (only the physical mass matrix and the free vibration mode shapes are required). The operations during simulation is thus reduced to multiplication with generalized coordinates.

A consistent loads equation, the force summation method for inertially coupled equations of motion, was derived based on the same underlying assumptions. For validation generalized loads are compared with respective loads contained in the EOM.

A practical test case was studied next. The influence of inertial coupling on local an integrated loads for where found to be relevant for hight angular rate / acceleration flight conditions. Especially very flexible structural components with large concentrated masses are influenced by inertial coupling terms.

The described set of equation EOM and FSM include important physical effects without requiring a different modelling strategy. This may be used to increase the precision of the dynamic simulation of loads recovery while adding a minimum on computational effort to uncoupled formulations. 


\section{Appendix}

\section{Generalization of the Inertia Tensor}

The inertia tensor for the deformed aircraft (16) used in the moment equation (32) will now be generalized. The inertia tensor is expanded to

$$
\begin{aligned}
\mathbf{J} & =\sum_{i} \mathbf{J}_{i}-\sum_{i} s k\left(\overline{\mathbf{r}}_{i}+\overline{\mathbf{d}}_{i}\right)^{2} m_{i} \\
& =\sum_{i} \mathbf{J}_{i}-\sum_{i}\left\{s k\left(\mathbf{s}_{i}\right)^{2}+s k\left(\mathbf{r}_{i}+\overline{\mathbf{d}}_{i}\right)^{2}+s k\left(\mathbf{s}_{i}\right) s k\left(\mathbf{r}_{i}+\overline{\mathbf{d}}_{i}\right)+s k\left(\mathbf{r}_{i}+\overline{\mathbf{d}}_{i}\right) s k\left(\mathbf{s}_{i}\right)\right\} m_{i} \\
& =\sum_{i} \mathbf{J}_{g, i}-\left(s k\left(\mathbf{r}_{i}+\overline{\mathbf{d}}_{i}\right)^{2}+s k\left(\mathbf{s}_{i}\right) s k\left(\mathbf{r}_{i}+\overline{\mathbf{d}}_{i}\right)+s k\left(\mathbf{r}_{i}+\overline{\mathbf{d}}_{i}\right) s k\left(\mathbf{s}_{i}\right)\right) m_{i}
\end{aligned}
$$

where the local inertia tensor w.r.t. the grid point $\mathbf{J}_{g, i}$ is directly available in the system mass matrix. Fully expansion yields:

$$
\begin{aligned}
& \mathbf{J}=\sum_{i} \mathbf{J}_{g, i}-(\underbrace{\sum_{i} s k\left(\mathbf{r}_{i}\right)^{2} m_{i}}_{A 1}+\underbrace{\sum_{i} s k\left(\mathbf{d}_{i}\right)^{2} m_{i}}_{A 2}+\underbrace{\sum_{i} s k\left(s k\left(\varphi_{i}\right) \mathbf{s}_{i}\right)^{2} m_{i}}_{A 3}+\underbrace{\sum_{i} s k\left(\mathbf{d}_{i}\right) s k\left(s k\left(\varphi_{i}\right) \mathbf{s}_{i}\right) m_{i}}_{A 4} \\
& +\underbrace{\sum_{i} s k\left(s k\left(\varphi_{i}\right) \mathbf{s}_{i}\right) s k\left(\mathbf{d}_{i}\right) m_{i}}_{A 5}+\underbrace{\sum_{i} s k\left(\mathbf{r}_{i}\right) s k\left(\mathbf{d}_{i}\right) m_{i}}_{A 6}+\underbrace{\sum_{i} s k\left(\mathbf{r}_{i}\right) s k\left(s k\left(\varphi_{i}\right) \mathbf{s}_{i}\right) m_{i}}_{A 7} \\
& +\underbrace{\sum_{i} s k\left(\mathbf{d}_{i}\right) s k\left(\mathbf{r}_{i}\right) m_{i}}_{A 8}+\underbrace{\sum_{i} s k\left(s k\left(\varphi_{i}\right) \mathbf{s}_{i}\right) s k\left(\mathbf{r}_{i}\right) m_{i}}_{A 9})
\end{aligned}
$$

The terms $A 1$ to $A 9$ of (59) will now be analyzed:

- A1 This constant term has the following elements

$$
A 1=\left[\begin{array}{ccc}
\sum_{i}\left(-\mathbf{r}_{i_{z}}^{2}-\mathbf{r}_{i_{y}}^{2}\right) & \sum_{i} \mathbf{r}_{i_{y}} \mathbf{r}_{i_{x}} & \sum_{i} \mathbf{r}_{i_{z}} \mathbf{r}_{i_{x}} \\
& \sum_{i}\left(-\mathbf{r}_{i_{z}}^{2}-\mathbf{r}_{i_{x}}^{2}\right) & \sum_{i} \mathbf{r}_{i_{z}} \mathbf{r}_{i_{y}} \\
\operatorname{sym} & & \sum_{i}\left(-\mathbf{r}_{i_{y}}^{2}-\mathbf{r}_{i_{x}}^{2}\right)
\end{array}\right] m_{i}
$$

- A2 The symmetric matrix $A 2$ in (59) can be cast into the form

$$
A 2=\left\langle\eta_{E}^{T} \widetilde{A 2}{ }_{j k} \eta_{E}\right\rangle
$$

where the notation $\langle\ldots\rangle$, shortening the summation over the matrix elements, is defined as follows:

$$
\begin{aligned}
\left\langle(\ldots)_{j k}\right\rangle & =\sum_{j=1}^{3} \sum_{k=1}^{3}(\ldots)_{j k} \mathbf{e}_{j} \mathbf{e}_{k}^{T} \\
\left\langle(\ldots)_{j}\right\rangle & =\sum_{j=1}^{3}(\ldots)_{j} \mathbf{e}_{j} \\
\text { with } \quad \mathbf{e}_{1} & =\left[\begin{array}{l}
1 \\
0 \\
0
\end{array}\right], \mathbf{e}_{2}=\left[\begin{array}{l}
0 \\
1 \\
0
\end{array}\right], \mathbf{e}_{3}=\left[\begin{array}{l}
0 \\
0 \\
1
\end{array}\right]
\end{aligned}
$$

The sub matrices $\widetilde{A 2}_{j k}$ in (1ㅡ) are as follows

$$
\begin{aligned}
& \widetilde{A 2}_{11}=\sum_{i}\left(-\boldsymbol{\Phi}_{g_{i_{z}} E_{t}}^{T} \boldsymbol{\Phi}_{g_{i_{z}} E_{t}}-\boldsymbol{\Phi}_{g_{i_{y}} E_{t}}^{T} \boldsymbol{\Phi}_{g_{i_{y}} E_{t}}\right) m_{i} \quad \widetilde{A 2}{ }_{12}=\sum_{i} \boldsymbol{\Phi}_{g_{i_{y}} E_{t}}^{T} \boldsymbol{\Phi}_{g_{i_{x}} E_{t}} m_{i} \\
& \widetilde{A 2}{ }_{13}=\sum_{i} \boldsymbol{\Phi}_{g_{i_{z}} E_{t}}^{T} \boldsymbol{\Phi}_{g_{i_{x}} E_{t}} m_{i} \quad \widetilde{A 2_{22}}=\sum_{i}\left(-\boldsymbol{\Phi}_{g_{i_{z}} E_{t}}^{T} \boldsymbol{\Phi}_{g_{i_{z}} E_{t}}-\boldsymbol{\Phi}_{g_{i_{x}} E_{t}}^{T} \boldsymbol{\Phi}_{g_{i_{x}} E_{t}}\right) m_{i} \\
& \widetilde{A 2}{ }_{23}=\sum_{i} \boldsymbol{\Phi}_{g_{i_{z}} E_{t}}^{T} \boldsymbol{\Phi}_{g_{i_{y}} E_{t}} m_{i} \quad \widetilde{A 2}{ }_{33}=\sum_{i}\left(-\boldsymbol{\Phi}_{g_{i_{y}} E_{t}}^{T} \boldsymbol{\Phi}_{g_{i_{y}} E_{t}}-\boldsymbol{\Phi}_{g_{i_{x}} E_{t}}^{T} \boldsymbol{\Phi}_{g_{i_{x}} E_{t}}\right) m_{i}
\end{aligned}
$$

- A3 The symmetric matrix $A 3$ in (59) is given by

$$
A 3=\sum_{i} s k\left(\left[\begin{array}{l}
\mathbf{a}_{1} \eta_{E} \\
\mathbf{a}_{2} \eta_{E} \\
\mathbf{a}_{3} \eta_{E}
\end{array}\right]\right)^{2} m_{i}
$$


With the row vectors

$$
\begin{aligned}
& \mathbf{a}_{1}=s_{z} \boldsymbol{\Phi}_{g_{i_{y}} E_{r}}-s_{y} \boldsymbol{\Phi}_{g_{i_{z}} E_{r}} \\
& \mathbf{a}_{2}=s_{x} \boldsymbol{\Phi}_{g_{i_{z}} E_{r}-s_{z} \boldsymbol{\Phi}_{g_{i_{x}} E_{r}}} \\
& \mathbf{a}_{1}=s_{y} \boldsymbol{\Phi}_{g_{i_{x}} E_{r}}-s_{z} \boldsymbol{\Phi}_{g_{i_{y}} E_{r}}
\end{aligned}
$$

resulting in a matrix of the following form

$$
A 3=\left\langle\eta_{E}^{T} \widetilde{A 3}_{j k} \eta_{E}\right\rangle
$$

where the sub matrices $\widetilde{A 3}_{j k}$ are as follows

$$
\begin{array}{lll}
\widetilde{A 2}_{11}=\sum_{i}\left(-\mathbf{a}_{3}^{T} \mathbf{a}_{3}-\mathbf{a}_{2}^{T} \mathbf{a}_{2}\right) m_{i} & \widetilde{A 2}_{12}=\sum_{i} \mathbf{a}_{2}^{T} \mathbf{a}_{1} m_{i} & \widetilde{A 2}_{13}=\sum_{i} \mathbf{a}_{3}^{T} \mathbf{a}_{1} m_{i} \\
\widetilde{A 2}_{21}=\widetilde{A 2}_{12}^{T} & \widetilde{A 2}_{22}=\sum_{i}\left(-\mathbf{a}_{3}^{T} \mathbf{a}_{3}-\mathbf{a}_{1}^{T} \mathbf{a}_{1}\right) m_{i} & \widetilde{A 2}_{23}=\sum_{i} \mathbf{a}_{3}^{T} \mathbf{a}_{2} m_{i} \\
\widetilde{A 2}_{31}=\widetilde{A 2}_{13}^{T} & \widetilde{A 2}_{32}=\widetilde{A 2}_{23} & \widetilde{A 2}{ }_{33}=\sum_{i}\left(-\mathbf{a}_{2}^{T} \mathbf{a}_{2}-\mathbf{a}_{1}^{T} \mathbf{a}_{1}\right) m_{i}
\end{array}
$$

- A4 The non symmetric matrix $A 4$ in (59) is given by

$$
A 4=\left\langle\eta_{E}^{T} \widetilde{A 4}{ }_{j k} \eta_{E}\right\rangle
$$

with the following sub matrices for $\widetilde{A 4}_{j k}$

$$
\begin{array}{rlrl}
\widetilde{A 4}_{11} & =\sum_{i}\left(-\boldsymbol{\Phi}_{g_{i_{z}} E_{t}}^{T} \mathbf{a}_{3}-\boldsymbol{\Phi}_{g_{i y} E_{t}}^{T} \mathbf{a}_{2}\right) m_{i} & \widetilde{A 4}_{12}=\sum_{i} \boldsymbol{\Phi}_{g_{i_{y}} E_{t}}^{T} \mathbf{a}_{1} m_{i} \\
\widetilde{A 4}_{13}=\sum_{i} \boldsymbol{\Phi}_{g_{i_{z}} E_{t}} \mathbf{a}_{1} m_{i} & \widetilde{A 4}_{21}=\sum_{i} \boldsymbol{\Phi}_{g_{i_{y}} E_{t}} \mathbf{a}_{2} m_{i} \\
\widetilde{A 4}_{22}=\sum_{i}\left(-\boldsymbol{\Phi}_{g_{i_{z}} E_{t}}^{T} \mathbf{a}_{3}-\boldsymbol{\Phi}_{g_{i_{x}} E_{t}}^{T} \mathbf{a}_{1}\right) m_{i} & \widetilde{A 4}_{23}=\sum_{i} \boldsymbol{\Phi}_{g_{i_{z}} E_{t}}^{T} \mathbf{a}_{2} m_{i} \\
\widetilde{A 4}_{31}=\sum_{i} \boldsymbol{\Phi}_{g_{i_{z}} E_{t}}^{T} \mathbf{a}_{1} m_{i} & \widetilde{A 4}_{32}=\sum_{i} \boldsymbol{\Phi}_{g_{i_{z}} E_{t}}^{T} \mathbf{a}_{2} m_{i} \\
\widetilde{A 4}_{33}=\sum_{i}\left(-\boldsymbol{\Phi}_{g_{i_{y}} E_{t}}^{T} \mathbf{a}_{2}-\boldsymbol{\Phi}_{g_{i_{x}} E_{t}}^{T} \mathbf{a}_{1}\right) m_{i} &
\end{array}
$$

- A5 The non symmetric matrix $A 5$ in (59) can be related to the term $A 4$ by

$$
A 5=\sum_{i} s k\left(s k\left(\varphi_{i}\right) \mathbf{s}_{i}\right) s k\left(\mathbf{d}_{i}\right) m_{i}=\sum_{i}\left(s k\left(\mathbf{d}_{i}\right) \operatorname{sk}\left(s k\left(\varphi_{i}\right) \mathbf{s}_{i}\right)\right)^{T} m_{i}=(A 4)^{T}
$$

Therefore $A 4+A 5$ again is a symmetric matrix

- A6 The non symmetric matrix $A 6$ in (59) is given by

$$
A 6=\widetilde{A 6}_{j k} \eta_{E}
$$

with the following row vectors for $\widetilde{A 6}_{j k}$

$$
\begin{array}{lll}
\widetilde{A 6}_{11}=\sum_{i}\left(-\mathbf{r}_{i_{z}} \boldsymbol{\Phi}_{\left.g_{i_{z}} E_{t}-\mathbf{r}_{i_{y}} \boldsymbol{\Phi}_{g_{i_{y}} E_{t}}\right) m_{i}}\right. & \widetilde{A 6}_{12}=\sum_{i} \mathbf{r}_{i_{y}} \boldsymbol{\Phi}_{g_{i_{x}} E_{t} m_{i}} \\
\widetilde{A 6}_{13}=\sum_{i} \mathbf{r}_{i_{z}} \boldsymbol{\Phi}_{g_{i_{x}} E_{t} m_{i}} & \widetilde{A 6}_{21}=\sum_{i} \mathbf{r}_{i_{x}} \boldsymbol{\Phi}_{g_{i_{y}} E_{t}} m_{i} \\
\widetilde{A 6}_{22}=\sum_{i}\left(-\mathbf{r}_{i_{z}} \boldsymbol{\Phi}_{g_{i_{z}} E_{t}}-\mathbf{r}_{i_{x}} \boldsymbol{\Phi}_{g_{i_{x}} E_{t}}\right) m_{i} & \widetilde{A 6}_{23}=\sum_{i} \mathbf{r}_{i_{z}} \boldsymbol{\Phi}_{g_{i_{y}} E_{t}} m_{i} \\
\widetilde{A 6}_{31}=\sum_{i} \mathbf{r}_{i_{x}} \boldsymbol{\Phi}_{g_{i_{z}} E_{t} m_{i}} & \widetilde{A 6}_{32}=\sum_{i} \mathbf{r}_{i_{y}} \boldsymbol{\Phi}_{g_{i_{z}} E_{t}} m_{i} \\
\widetilde{A 6}_{33}=\sum_{i}\left(-\mathbf{r}_{i_{y}} \boldsymbol{\Phi}_{g_{i_{y}} E_{t}}-\mathbf{r}_{i_{x}} \boldsymbol{\Phi}_{g_{i_{x}} E_{t}}\right) m_{i} &
\end{array}
$$

- A7 The non symmetric matrix $A 7$ in (59) is given by

$$
A 7=\widetilde{A 7}_{j k} \eta_{E}
$$

with the following row vectors for $\widetilde{A 7}_{j k}$

$$
\begin{aligned}
& \widetilde{A 7}_{11}=\sum_{i}\left(-\mathbf{r}_{i_{z}} \mathbf{a}_{3}-\mathbf{r}_{i_{y}} \mathbf{a}_{2}\right) m_{i} \quad \widetilde{A 7}_{12}=\sum_{i} \mathbf{r}_{i_{y}} \mathbf{a}_{1} m_{i} \\
& \widetilde{A 7}_{13}=\sum_{i} \mathbf{r}_{i_{z}} \mathbf{a}_{1} m_{i} \quad \widetilde{A 7}_{21}=\sum_{i} \mathbf{r}_{i_{x}} \mathbf{a}_{2} m_{i} \\
& \widetilde{A 7}_{22}=\sum_{i}\left(-\mathbf{r}_{i_{z}} \mathbf{a}_{3}-\mathbf{r}_{i_{x}} \mathbf{a}_{1}\right) m_{i} \quad \widetilde{A 7}_{23}=\sum_{i} \mathbf{r}_{i_{z}} \mathbf{a}_{2} m_{i} \\
& \widetilde{A 7}_{31}=\sum_{i} \mathbf{r}_{i_{x}} \mathbf{a}_{3} m_{i} \quad \widetilde{A 7}_{32}=\sum_{i} \mathbf{r}_{i_{y}} \mathbf{a}_{3} m_{i} \\
& \widetilde{A 7}_{33}=\sum_{i}\left(-\mathbf{r}_{i_{y}} \mathbf{a}_{2}-\mathbf{r}_{i_{x}} \mathbf{a}_{1}\right) m_{i}
\end{aligned}
$$

18 of 21 
- A8 The non symmetric matrix $A 8$ in (59) is given by

$$
A 8=(A 6)^{T}
$$

Therefore $A 6+A 8$ again is a symmetric matrix

- A9 The non symmetric matrix $A 9$ in (59) is given by

$$
A 9=(A 7)^{T}
$$

Therefore $A 7+A 9$ again is a symmetric matrix

Inserting the terms $A 1(\underline{60})$ to $A 9(\underline{71})$ in equation (59) and using

$$
\begin{aligned}
\widetilde{B}_{j k} & =\widetilde{A 2}_{j k}+\widetilde{A 3}_{j k}+\widetilde{A 4}_{j k}+\widetilde{A 5}_{j k} \\
\widetilde{C}_{j k} & =\widetilde{A 6}_{j k}+\widetilde{A 7}_{j k} \\
\widetilde{D}_{j k} & =\widetilde{A 8}_{j k}+\widetilde{A 9}_{j k}
\end{aligned}
$$

yields the final expression for the inertia tensor $\mathbf{J}$ :

$$
\mathbf{J}=\sum_{i} \mathbf{J}_{g, i}-A 1-\left[\begin{array}{ccc}
\eta_{E}^{T} \widetilde{B}_{11} \eta_{E} & \eta_{E}^{T} \widetilde{B}_{12} \eta_{E} & \eta_{E}^{T} \widetilde{B}_{13} \eta_{E} \\
& \eta_{E}^{T} \widetilde{B}_{22} \eta_{E} & \eta_{E}^{T} \widetilde{B}_{23} \eta_{E} \\
\operatorname{sym} & & \eta_{E}^{T} \widetilde{B}_{33} \eta_{E}
\end{array}\right]-\left[\begin{array}{cccc}
\eta_{E}^{T} \widetilde{C}_{11}+\widetilde{D}_{11} \eta_{E} & \eta_{E}^{T} \widetilde{C}_{12}+\widetilde{D}_{12} \eta_{E} & \eta_{E}^{T} \widetilde{C}_{13}+\widetilde{D}_{13} \eta_{E} \\
& \eta_{E}^{T} \widetilde{C}_{22}+\widetilde{D}_{22} \eta_{E} & \eta_{E}^{T} \widetilde{C}_{23}+\widetilde{D}_{23} \eta_{E} \\
\operatorname{sym} & & \eta_{E}^{T} \widetilde{C}_{33}+\widetilde{D}_{33} \eta_{E}
\end{array}\right]
$$

It may also be written in a compact form

$$
\mathbf{J}=\sum_{i} \mathbf{J}_{g, i}-A 1-\left\langle\eta_{E}^{T} \widetilde{B}_{j k} \eta_{E}\right\rangle-\left\langle\eta_{E}^{T} \widetilde{C}_{j k}+\widetilde{D}_{j k} \eta_{E}\right\rangle
$$

The time derivative $\stackrel{\circ}{\mathbf{J}}$ can be easily obtained from $(\overline{75})$ since $\sum_{i} \mathbf{J}_{g, i}, A 1, \widetilde{B}_{j k}, \widetilde{C}_{j k}, \widetilde{D}_{j k}$ are no functions of time. Hence $\stackrel{\circ}{\mathbf{J}}$ is

$$
\stackrel{\circ}{\mathbf{J}}=-\left\langle\stackrel{\circ}{\eta}_{E}^{T} \widetilde{B}_{j k} \eta_{E}\right\rangle-\left\langle\eta_{E}^{T} \widetilde{B}_{j k} \stackrel{\circ}{\eta}_{E}\right\rangle-\left\langle\stackrel{\circ}{\eta}_{E}^{T} \widetilde{C}_{j k}+\widetilde{D}_{j k} \stackrel{\circ}{\eta}_{E}\right\rangle
$$

The preceding equations for the inertia tensor and its time derivative are fully generalized, all physical values where expressed by modal coordinates.

\section{Generalization of the $\mathrm{h}$ - Term}

Next the term $\mathbf{h}$ defined in the moment equation $\left(\underline{32)}\right.$ will be generalized. $\mathbf{h}=\sum_{i}\left(\overline{\mathbf{d}}_{i} \times \stackrel{\circ}{\mathbf{d}_{i}}\right) m_{i}+\sum_{i} \mathbf{J}_{i} \stackrel{\circ}{\varphi_{i}}$ with $\overline{\mathbf{d}}_{i}=\mathbf{d}_{i}+\varphi_{i} \times \mathbf{s}_{i}$ and $\stackrel{\circ}{\mathbf{d}}_{i}=\stackrel{\circ}{\mathbf{d}}_{i}+\stackrel{\circ}{\varphi}_{i} \times \mathbf{s}_{i}$ the term can be expanded to

$$
\mathbf{h}=\underbrace{\sum_{i} \mathbf{d}_{i} \times \stackrel{\circ}{\mathbf{d}}_{i} m_{i}}_{h 1}+\underbrace{\sum_{i} \mathbf{d}_{i} \times\left(\stackrel{\circ}{\varphi}_{i} \times \mathbf{s}_{i}\right) m_{i}}_{h 2}+\underbrace{\sum_{i}\left(\varphi_{i} \times \mathbf{s}_{i}\right) \times \stackrel{\circ}{\mathbf{d}}_{i} m_{i}}_{h 3}+\underbrace{\sum_{i}\left(\varphi_{i} \times \mathbf{s}_{i}\right) \times\left(\stackrel{\circ}{\varphi}_{i} \times \mathbf{s}_{i}\right) m_{i}}_{h 4}+\underbrace{\sum_{i} \mathbf{J}_{i} \stackrel{\circ}{\varphi}_{i}}_{h 5}
$$

Expansion of the preceding expression for $h 1$ yields

$$
h 1=\left[\begin{array}{lll}
\eta_{E}^{T} \sum_{i}\left(-\boldsymbol{\Phi}_{g_{i_{z}} E_{t}}^{T} \boldsymbol{\Phi}_{g_{i_{y}} E_{t}}+\boldsymbol{\Phi}_{g_{i_{y}} E_{t}}^{T} \boldsymbol{\Phi}_{g_{i_{z}} E_{t}}\right) m_{i} & \stackrel{\circ}{\eta}_{E} \\
\eta_{E}^{T} \sum_{i}\left(+\boldsymbol{\Phi}_{g_{i_{z}} E_{t}}^{T} \boldsymbol{\Phi}_{g_{i_{x}} E_{t}}-\boldsymbol{\Phi}_{g_{i_{x}} E_{t}}^{T} \boldsymbol{\Phi}_{g_{i_{z}} E_{t}}\right) m_{i} & \stackrel{\circ}{\eta}_{E} \\
\eta_{E}^{T} \sum_{i}\left(-\boldsymbol{\Phi}_{g_{i_{y}} E_{t}}^{T} \boldsymbol{\Phi}_{g_{i_{x}} E_{t}}+\boldsymbol{\Phi}_{g_{i_{x}} E_{t}}^{T} \boldsymbol{\Phi}_{g_{i_{y}} E_{t}}\right) m_{i} & {\stackrel{\circ}{\eta_{E}}}^{T}
\end{array}\right]=\left\langle\eta_{E}^{T} \widetilde{h 1_{j}}{\left.\stackrel{\circ}{\eta_{E}}\right\rangle}\right.
$$

note that $\widetilde{h 1}_{j}=-\widetilde{h 1}_{j}^{T}$. The expanded term $h 2$ may be expressed by

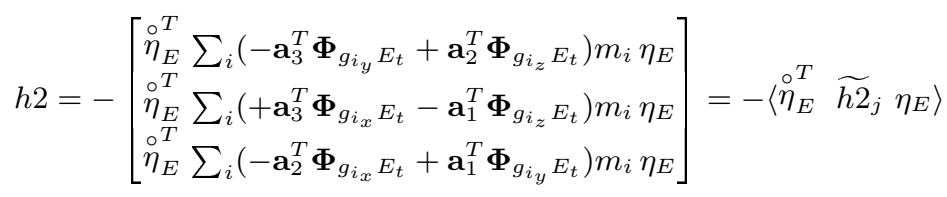


The term $h 3$ can be written in a similar structure:

$$
h 3=\left\langle\eta_{E}^{T} \widetilde{h 3_{j}} \stackrel{\circ}{\eta}_{E}\right\rangle \quad \text { with } \quad \widetilde{h 3}_{j}=\widetilde{h 2}_{j}
$$

Expansion of the expression for $h 4$ yields

$$
h 4=\left\langle\eta_{E}^{T} \widetilde{h 4}_{j} \stackrel{\circ}{\eta}_{E}\right\rangle
$$

with

$$
\begin{aligned}
& \widetilde{h 4_{1}}=\sum_{i}\left[\left(\boldsymbol{\Phi}_{g_{i_{z}} E_{r}}^{T} \mathbf{s}_{x}-\boldsymbol{\Phi}_{g_{i_{x}} E_{r}}^{T} \mathbf{s}_{z}\right)\left(\boldsymbol{\Phi}_{g_{i_{x}} E_{r}} \mathbf{s}_{y}-\boldsymbol{\Phi}_{g_{i_{y}} E_{r}} \mathbf{s}_{x}\right)-\left(\boldsymbol{\Phi}_{g_{i_{x}} E_{r}}^{T} \mathbf{s}_{y}-\boldsymbol{\Phi}_{g_{i_{y}} E_{r}}^{T} \mathbf{s}_{x}\right)\left(\boldsymbol{\Phi}_{g_{i_{z}} E_{r}} \mathbf{s}_{x}-\boldsymbol{\Phi}_{g_{i_{x}} E_{r}} \mathbf{s}_{z}\right)\right] m_{i} \\
& \widetilde{h 4_{2}}=\sum_{i}\left[\left(\boldsymbol{\Phi}_{g_{i x} E_{r}}^{T} \mathbf{s}_{y}-\boldsymbol{\Phi}_{g_{i_{y}} E_{r}}^{T} \mathbf{s}_{x}\right)\left(\boldsymbol{\Phi}_{g_{i} E_{r}} \mathbf{s}_{z}-\boldsymbol{\Phi}_{g_{i_{z}} E_{r}} \mathbf{s}_{y}\right)-\left(\boldsymbol{\Phi}_{g_{i_{y}} E_{r}}^{T} \mathbf{s}_{z}-\boldsymbol{\Phi}_{g_{i_{z}} E_{r}}^{T} \mathbf{s}_{y}\right)\left(\boldsymbol{\Phi}_{g_{i_{x}} E_{r}} \mathbf{s}_{y}-\boldsymbol{\Phi}_{g_{i_{y}} E_{r}} \mathbf{s}_{x}\right)\right] m_{i} \\
& \widetilde{h 4}_{3}=\sum_{i}\left[\left(\boldsymbol{\Phi}_{g_{i} E_{r}}^{T} \mathbf{s}_{z}-\boldsymbol{\Phi}_{g_{i_{z}} E_{r}}^{T} \mathbf{s}_{y}\right)\left(\boldsymbol{\Phi}_{g_{i_{z}} E_{r}} \mathbf{s}_{x}-\boldsymbol{\Phi}_{g_{i_{x}} E_{r}} \mathbf{s}_{z}\right)-\left(\boldsymbol{\Phi}_{g_{i_{z}} E_{r}}^{T} \mathbf{s}_{x}-\boldsymbol{\Phi}_{g_{i_{x}} E_{r}}^{T} \mathbf{s}_{z}\right)\left(\boldsymbol{\Phi}_{g_{i_{y}} E_{r}} \mathbf{s}_{z}-\boldsymbol{\Phi}_{g_{i_{z}} E_{r}} \mathbf{s}_{y}\right)\right] m_{i}
\end{aligned}
$$

note that $\widetilde{h 4}_{j}=-\widetilde{h 4}_{j}^{T}$. The term $h 5$ can be written as follows $\sum_{i} \mathbf{J}_{i} \stackrel{\circ}{\varphi}_{i}=\sum_{i} \mathbf{J}_{i} \boldsymbol{\Phi}_{g_{i} E_{r}} \stackrel{\circ}{\eta}_{E}=\widetilde{h 5} \stackrel{\circ}{\eta}_{E}$. With the preceding expressions the $\mathbf{h}$-term and the time derivative $\stackrel{\circ}{\mathbf{h}}$ can finally be written in the following form

$$
\begin{aligned}
& \mathbf{h}=-\left\langle\stackrel{\circ}{\eta}_{E}^{T} \widetilde{h 2}_{j} \eta_{E}\right\rangle+\left\langle\eta_{E}^{T}\left(\widetilde{h 1}_{j}+\widetilde{h 2}_{j}+\widetilde{h 4}_{j}\right) \stackrel{\circ}{\eta}_{E}\right\rangle+\widetilde{h 5} \stackrel{\circ}{\eta}_{E} \\
& \stackrel{\circ}{\mathbf{h}}=\left\langle\stackrel{\circ}{\eta}_{E}^{T}\left(\widetilde{h 1}_{j}+\widetilde{h 4}_{j}\right) \stackrel{\circ}{\eta}_{E}\right\rangle-\left\langle\stackrel{\circ T}{\eta}_{E} \widetilde{h 2}_{j} \eta_{E}\right\rangle+\left\langle\eta_{E}^{T}\left(\widetilde{h 1}_{j}+\widetilde{h 2}_{j}+\widetilde{h 4}_{j}\right) \stackrel{\circ}{\eta}_{E}\right\rangle+\widetilde{h 5} \stackrel{\circ}{\eta}_{E}
\end{aligned}
$$

\section{Validation of Modal Form}

The generalized form of the coupling terms is now validated by comparing it with the physical form. All modal coupling components are included in the modal form of the inertia tensor $(\mathbf{7 5})$ and h-term $(\mathbf{8 2})$. Therefore the physical form of the inertia tensor $(\underline{16})$ and h-term $(\underline{33})$ can be used to validate the generalization process.

A generic aileron input is used as a test case, since it excites all of the elastic mode shapes. Both, physical and modal forms are implemented in a common simulation environment. Fig 11 depicts the time response of the h-term for each component and the difference between the physical and modal form. The modal form of the h-term yields the same results as the physical form. Difference are of the order of the numerical precision.
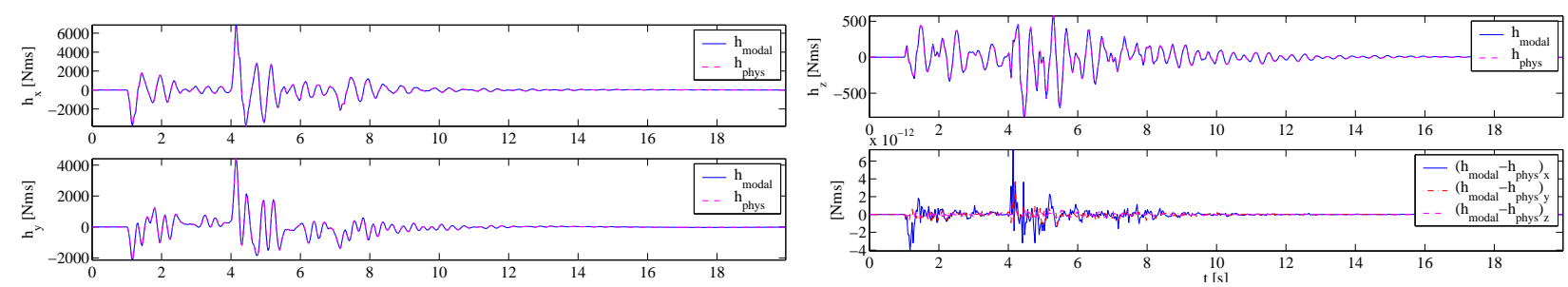

Figure 11. Comparison of physical an modal implementation of h-term

\section{References}

${ }^{1}$ E. Albano and W.P. Rodden. A doublet-lattice method for calculating lift distributions on oscillating surfaces in subsonic flows. Journal of Aircraft, 7(2):279-285, 1969.

${ }^{2}$ K.J. Bathe. Finite Element Procedures. Prentice Hall, 1996.

${ }^{3}$ R. L. Bisplinghoff, H. Ashley, and R. L. Halfman. Aeroelasticity. Dover Publications, Inc., 1955.

${ }^{4}$ C.S. Buttrill, T.A. Zeiler, and P.D. Arbuckle. Nonlinear simulation of a flexible aircraft in maneuvering flight. In $A I A A$ Flight Simulation Technologies Conference, number AIAA 87-2501. AIAA, 1987.

${ }^{5}$ J. R. Canavin and P. W. Likins. Floating reference frames for flexible spacecraft. Journal of Spacecraft and Rockets, 14(12):724-732, December 1977.

${ }^{6}$ R. K. Cavin and A. R. Dusto. Hamilton's principle: Finite-element methods and flexible body dynamics. AIAA Journal, 15(12):1684-1690, September 1977.

${ }^{7}$ R. R. Craig. Structural Dynamics. Wiley, 1981.

${ }^{8}$ C.Reschke. Berechnung dynamischer lasten bei elastischen strukturen. Technical Report Diplomarbeit, Universität Stuttgart, 2003.

${ }^{9} \mathrm{~F}$. Engelsen and E. Livne. Mode acceleration based random gust stresses in aeroservoelastic optimization. Journal of Aircraft, 41(2):335-347, 2004.

${ }^{10}$ B. Etkin. Dynamics of Flight. John Wiley \& Sons, INC, 1996.

${ }^{11}$ S.H.J.A. Fransen. An overview and comparison of otm formulations on the basis of the mode displacement method and the mode acceleration method. 2001. 
${ }^{12}$ M. Hanel. Robust Integrated Flight and Aeroelastic Control System Design for a Large Transport Aircraft. PhD thesis, Universität Stuttgart, 2001.

${ }^{13}$ W. Hauger, W.Schnell, and D. Gross. Technische Mechanik 3. Springer-Verlag, 1989.

${ }^{14} \mathrm{M}$. Karpel and E. Presente. Structural dynamic loads in response to impulsive excitation. In International Forum on Aeroelasticity and Structural Dynamics, pages 1059-1075, May 1993.

${ }^{15}$ M.J.Brenner K.K.Gupta and L.S.Voelker. Development of an integrated aeroservoelastic analysis program and correlation with test data. Technical report, 1991.

${ }^{16}$ D. McLean. Automatic Filght Control Systems. Prentice Hall, 1990.

${ }^{17}$ L. Meirovitch. Methods of Analytical Dynamics. McGraw-Hill Book Company, 1970.

${ }^{18} \mathrm{~L}$. Meirovitch. Hybrid state equations of motion for flexible bodies in terms of quasi-coordinates. Journal of Guidance, 14(5):1008-1013, 1988.

${ }^{19} \mathrm{~L}$. Meirovitch and I. Tuzcu. Time simulation of the response of maneuvering flexible aircraft. Jounal of Guidance, Control and Dynamics, 27(5):814-828, September-October 2004.

${ }^{20}$ M.Karpel. Reduced-order models for integrated aeroservoelastic optimization. Journal of Aircraft, 36(1):146-155, 1999.

${ }^{21}$ A. S. Pototzky. New and existing techniques for dynamic loads analysis of flexible airplanes. Journal of Aircraft, 23(4):340-347, 1985.

${ }^{22}$ K. L. Roger. Airplane math modeling methods for active control design. In AGARD Structures and Materials Panel, number AGARD/CP-228, pages 4-1 - 4-11. AGARD, April 1977.

${ }^{23}$ L. v. Schmidt. Introduction to Aircraft Flight Dynamics. AIAA Education Series, 1998.

${ }^{24}$ M. R. Waszak and D. K. Schmidt. Flight dynamics of aeroelastic vehicles. Journal of Aircraft, 25(6):563-571, 1988. 